DMUS-MP-13/16

\title{
Index theory and dynamical symmetry enhancement near IIB horizons
}

\author{
U. Gran ${ }^{1}$, J. Gutowski ${ }^{2}$ and G. Papadopoulos ${ }^{3}$ \\ ${ }^{1}$ Fundamental Physics \\ Chalmers University of Technology \\ SE-412 96 Göteborg, Sweden \\ ${ }^{2}$ Department of Mathematics \\ University of Surrey \\ Guildford, GU2 7XH, UK \\ ${ }^{3}$ Department of Mathematics \\ King's College London \\ Strand \\ London WC2R 2LS, UK
}

\begin{abstract}
We show that the number of supersymmetries of IIB black hole horizons is $N=2 N_{-}+$ 2 index $\left(D_{\lambda}\right)$, where index $\left(D_{\lambda}\right)$ is the index of the Dirac operator twisted with the line bundle $\lambda^{\frac{1}{2}}$ of IIB scalars, and $N_{-}$is the dimension of the kernel of a horizon Dirac operator which depends on IIB fluxes. Therefore, all IIB horizons preserve an even number of supersymmetries. In addition if the horizons have non-trivial fluxes and $N_{-} \neq 0$, then index $\left(D_{\lambda}\right) \geq 0$ and the horizons admit an $\mathfrak{s l}(2, \mathbb{R})$ symmetry subalgebra. This provides evidence that all such horizons have an AdS/CFT dual. Furthermore if the orbits of $\mathfrak{s l}(2, \mathbb{R})$ are two-dimensional, the IIB horizons are warped products $A d S_{2} \times{ }_{w} \mathcal{S}$.
\end{abstract}




\section{Introduction}

It has been known for some time that black holes and branes exhibit symmetry enhancement near their horizons $[1,2,3]$. Typically, this enhancement leads to a superconformal symmetry which in turn has been instrumental in the AdS/CFT correspondence [4] and black hole entropy counting $[5,6]$. Originally, this symmetry enhancement has been observed on an either a case by case basis or under some additional symmetry assumptions, see [7] for a recent review and references within, but more recently it has been shown that it is a general phenomenon. So far a proof has been given for odd-dimensional black hole horizons ${ }^{1}$ like those of minimal 5-dimensional gauged supergravity and of M-theory $[8,9]$. It relies on the smoothness of horizons, the compactness of the horizon sections, Lichnerowicz type theorems, and the vanishing of the index of the Dirac operator on odd dimensional manifolds. In particular, one shows first that there is an enhancement of supersymmetry and then that the horizons with non-trivial fluxes admit an $\mathfrak{s l}(2, \mathbb{R})$ symmetry subalgebra. In turn, this can be seen as evidence that all such horizons have an AdS/CFT dual.

The investigation of the symmetry enhancement of IIB horizons is expected to be different from that of M-horizons for two different reasons. First, the index of the Dirac operator may not vanish on even-dimensional manifolds. Second, IIB and the majority of other supergravity theories apart from the gravitino Killing spinor equation (KSE) also have additional KSEs associated with the rest of fermions in the spectrum. Because of this, the Lichnerowicz type theorems needed to show symmetry enhancement must be generalized, so that all the Killing spinors, and not only the solutions of the gravitino KSE, are in a one to one correspondence with the zero modes of a Dirac operator.

In this paper, we shall show that the number of Killing spinors of IIB horizons is given by

$$
N=2 N_{-}+2 \operatorname{index}\left(D_{\lambda}\right),
$$

where $N_{-}$is the dimension of the kernel of a Dirac operator $\mathcal{D}^{(-)}$on the horizon section $\mathcal{S}$ which depends on the IIB form fluxes, and $D_{\lambda}$ is the twisted Dirac operator on $\mathcal{S}$ with respect to the $\lambda^{\frac{1}{2}}$ line bundle which arises in the description of IIB scalars. In particular,

$$
\operatorname{index}\left(D_{\lambda}\right)=\hat{A} \operatorname{ch}\left(\lambda^{\frac{1}{2}}\right)[\mathcal{S}]=\frac{1}{5760}\left(-4 p_{2}+7 p_{1}^{2}\right)-\frac{1}{192} p_{1} c_{1}^{2}+\frac{1}{384} c_{1}^{4},
$$

where $\hat{A}$ is a A-roof genus, $\operatorname{ch}\left(\lambda^{\frac{1}{2}}\right)$ is the Chern character of $\lambda^{\frac{1}{2}}, p_{1}$ and $p_{2}$ are the first and second Pontryagin classes of $\mathcal{S}$, and $c_{1}$ is the first Chern class of $\lambda$. In many examples ${ }^{2}$ $c_{1}=0$ and the above formula gives the index of the standard Dirac operator for 8dimensional spin manifolds.

\footnotetext{
${ }^{1}$ The black hole horizons in this paper are assumed to be Killing horizons with compact horizon sections. Such horizons can be event horizons for black holes, and brane configurations which after intersections and wrappings behave effectively as 0-branes. This justifies the requirement that the horizon section $\mathcal{S}$ is compact without boundary because $\mathcal{S}$ is thought of as the space that surrounds the point-like object. The associated black hole and brane solutions are not necessarily taken to be asymptotically flat.

${ }^{2}$ If the IIB scalars take values in the hyperbolic upper half plane, then $c_{1}=0$ as it is a contractible space. See section 5 for the case where the scalars take values in the fundamental domain of the modular group.
} 
An immediate consequence of the formula (1.1) is that the number of supersymmetries preserved by IIB horizons is even. Moreover, there are two cases to consider depending on whether $N_{-}$vanishes or not. If $N_{-} \neq 0$, one can show that $\operatorname{index}\left(D_{\lambda}\right) \geq 0$ and that the near horizon geometries with non-trivial fluxes admit an $\mathfrak{s l}(2, \mathbb{R})$ symmetry subalgebra. In addition, the vector fields which generate the $\mathfrak{s l}(2, \mathbb{R})$ symmetry have either 2- or 3dimensional orbits. If the orbits are 2-dimensional, then the near horizon geometry is a warped product of $A d S_{2}$ with the horizon section $\mathcal{S}, A d S_{2} \times{ }_{w} \mathcal{S}$. This extends the results we have obtained on symmetry enhancement for 5-dimensional and M-horizons to IIB horizons.

On the other hand if $N_{-}=0$, then the number of supersymmetries preserved is expressed in terms of the index of the Dirac operator. The geometry of such horizons has already been investigated in [18]. The formula for $N$ in this case resembles that for the number of parallel spinors on 8-dimensional manifolds $M$ with (torsion-free) holonomy strictly $\operatorname{Spin}(7), S U(4), S p(2)$ and $S p(1) \times S p(1)$. In particular, it is known that the number of parallel spinors $N_{p}$ is given in terms of the index [10] of the Dirac operator ${ }^{3}$ as

$$
N_{p}=\operatorname{index}(D)=\hat{A}[M],
$$

for $N_{p}=1,2,3$ and 4, respectively. Therefore $N_{p}$ is a topological invariant. In turn, (1.3) can be used to test whether a manifold with known first and second Pontryagin numbers can admit a metric for which the Levi-Civita connection has holonomy one of the above four groups.

The formula (1.1) also provides a topological restriction on the horizon sections. We know that $N \leq 32$. As a result, one can conclude that index $\left(D_{\lambda}\right) \leq 16-N_{-}$with $0 \leq N_{-} \leq 16$. So for all horizon sections $0 \leq \operatorname{index}\left(D_{\lambda}\right) \leq 16$. Furthermore since there are no IIB backgrounds with strictly 29, 30, 31 supersymmetries [12] and the unique solution [13] with 28 supersymmetries [14] does not satisfy the compactness restrictions of near horizon geometries, one also has that $N_{-}+\operatorname{index}\left(D_{\lambda}\right) \neq 14,15$.

It is well known that IIB supergravity consistently truncates to the common sector and to gravity coupled only to the 5 -form flux. In both cases $c_{1}=0$. We show that common sector horizons always preserve an even number of supersymmetries and that the index of the Dirac operator vanishes on the horizon sections. Moreover, the geometry of all common sector horizons can be understood in terms of that of heterotic horizons [24]. In particular, for all common sector horizons, the orbit of the $\mathfrak{s l}(2, \mathbb{R})$ symmetry is always 3-dimensional - there are no common sector horizons which are warped $\operatorname{Ad} S_{2}$ products.

Turning to horizons with only 5 -form flux, there are two cases to consider depending on whether $N_{-}$vanishes. If $N_{-} \neq 0$, and an additional mild assumption when the orbit of $\mathfrak{s l}(2, \mathbb{R})$ is 3 -dimensional, all such horizons preserve $4 \mathrm{k}$ supersymmetries and the index of the Dirac operator vanishes on the horizon sections. As a consequence the $\hat{A}$-genus of horizon sections vanishes. On the other hand if $N_{-}=0$, the number of supersymmetries

\footnotetext{
${ }^{3}$ On 8-dimensional manifolds, the vanishing of the topological obstruction $8 e-4 p_{2}+p_{1}^{2}=0$ for the existence of a $\operatorname{Spin}(7)$ structure and the expression for the signature $45 \sigma=7 p_{2}-p_{1}^{2}$ allows one to write index $(D)=\hat{A}[M]=\frac{1}{5760}\left(-4 p_{2}+7 p_{1}^{2}\right)=\frac{1}{24}\left(-1+b_{1}-b_{2}+b_{3}+b_{4}^{+}-2 b_{4}^{-}\right)$[11], where $b_{i}$ are the Betti numbers, $b_{4}^{ \pm}$are the number of self-dual and anti-self-dual Harmonic 4 -forms, $\sigma=b_{4}^{+}-b_{4}^{-}$and $e=2-2 b_{1}+2 b_{2}-2 b_{3}+b_{4}$ is the Euler number.
} 
preserved is a given in terms of the index of a Dirac operator and so it is a topological invariant. The geometry of such horizons has been investigated in [15].

The proof of our results relies on first integrating the KSEs of IIB supergravity along the lightcone directions after decomposing the Killing spinor as $\epsilon=\epsilon_{+}+\epsilon_{-}$using the lightcone projectors $\Gamma_{ \pm} \epsilon_{ \pm}=0$, and then showing that the independent KSEs are those that are obtained by the naive restriction of IIB KSEs on the horizon section $\mathcal{S}$. The integration along the lightcone directions ${ }^{4}$ gives rise to two horizon supercovariant connections $\nabla^{( \pm)}$on the sections $\mathcal{S}$, and two associated horizon Dirac operators $\mathcal{D}^{( \pm)}$. Furthermore, it establishes a map $\Gamma_{+} \Theta_{-}$, which depends on IIB fluxes, from the $\epsilon_{-}$Killing spinors to the $\epsilon_{+}$Killing spinors. The proof proceeds with the demonstration of two Lichnerowicz type theorems, one for each $\mathcal{D}^{( \pm)}$horizon Dirac operator. These are established with the use of the (strong) maximum principle for the $\mathcal{D}^{(+)}$operator and with a partial integration argument for $\mathcal{D}^{(-)}$. In both cases, the proof requires the field equations and Bianchi identities of IIB supergravity. The novelty in the proof of these two Lichnerowicz type theorems is that in addition to implying that the zero modes of $\mathcal{D}^{( \pm)}$are $\nabla^{( \pm)}$-parallel, they also establish that these zero modes also solve the two algebraic horizon KSEs associated with the IIB dilatino KSE. Then the formula (1.1) is shown as an application of the index theorem for the Dirac operator after observing that the number of Killing spinors is $N=N_{+}+N_{-}$, where $N_{ \pm}=\operatorname{dim} \operatorname{Ker} \mathcal{D}^{( \pm)}$, and demonstrating that the kernel of adjoint of $\mathcal{D}^{(+)}$can be effectively identified with that of $\mathcal{D}^{(-)}$.

The proof of $\mathfrak{s l}(2, \mathbb{R})$ symmetry for horizons with $N_{-} \neq 0$ utilizes the linear map $\Gamma_{+} \Theta_{-}$ mentioned above. In particular we show that for horizons with non-trivial fluxes, $\Gamma_{+} \Theta_{-}$ is an injection and so for each zero mode of $\mathcal{D}^{(-)}$there is a zero mode of $\mathcal{D}^{(+)}$. Using such a pair of zero modes, one can construct three 1-form Killing spinor bi-linears and demonstrate that satisfy an $\mathfrak{s l}(2, \mathbb{R})$ algebra.

This paper has been organized as follows. In section 2, we integrate the KSEs along the lightcone directions and find the independent KSEs. In section 3, we outline the proof of two Lichnerowicz type theorems for the horizon Dirac operators. In section 4 , we prove (1.1). In section 5, we relate the zero modes of the two horizon Dirac operators and in section 6 we use this result to demonstrate the $\mathfrak{s l}(2, \mathbb{R})$ symmetry of some IIB horizons. In section 7 , we investigate the common sector and IIB horizons with only 5 -form fluxes, and in section 8 we give our conclusions and state two conjectures. In appendix A, we summarize the field equations and Bianchi identities we use through this paper, and in appendices $\mathrm{B}$ and $\mathrm{C}$ we provide detailed proofs of the theorems we have used. In appendix $\mathrm{D}$ a proof of the fact that the Killing vectors constructed from Killing spinors preserve the fluxes up to a $U(1)$ transformation is given.

\footnotetext{
${ }^{4}$ To integrate the KSEs we do not use the bilinear matching condition which has been extensively applied to investigate near horizon geometries following [16] but which imposes an additional restriction on the solutions, see also [17].
} 


\section{Horizon fields and KSEs}

\subsection{Horizon fields, Bianchi identities and field equations}

The fields of IIB supergravity near an extreme horizon can be expressed [18] as

$$
\begin{aligned}
d s^{2} & =2 \mathbf{e}^{+} \mathbf{e}^{-}+\delta_{i j} \mathbf{e}^{i} \mathbf{e}^{j}, \quad F=r \mathbf{e}^{+} \wedge X+\mathbf{e}^{+} \wedge \mathbf{e}^{-} \wedge Y+\star_{8} Y, \\
G & =r \mathbf{e}^{+} \wedge L+\mathbf{e}^{+} \wedge \mathbf{e}^{-} \wedge \Phi+H, \quad P=\xi
\end{aligned}
$$

where we have introduced the frame

$$
\mathbf{e}^{+}=d u, \quad \mathbf{e}^{-}=d r+r h-\frac{1}{2} r^{2} \Delta d u, \quad \mathbf{e}^{i}=e_{I}^{i} d y^{I},
$$

and the self-duality of $F$ requires that $X=-\star_{8} X$. The dependence on the coordinates $u$ and $r$ is explicitly given, and the horizon section $\mathcal{S}$ is the co-dimension 2 submanifold $r=u=0 . \Delta, h, Y$ are 0 -, 1 - and 3-forms on $\mathcal{S}$, respectively, $\Phi, L$ and $H$ are $\lambda$-twisted 1 -, 2- and 3 -forms on $\mathcal{S}$, respectively, and $\xi$ is a $\lambda^{2}$-twisted 1-form on $\mathcal{S}$, where $\lambda$ arises from the pull back of the canonical bundle on the scalar manifold $S U(1,1) / U(1)$ on $\mathcal{S}$. Throughout, we require that all field strengths are taken to be at least $C^{2}$ differentiable on $\mathcal{S}$. This is one of our key assumptions. Furthermore, we shall not dwell on whether we consider $S U(1,1) / U(1)$ or the fundamental domain of the modular group as the IIB scalars manifold, however see section 5 .

Substituting the fields (2.1) into the Bianchi identities and field equations of IIB supergravity, one finds that $L$ and $X$ can be expressed in terms of other fields. The remaining Bianchi identities and field equations are summarized in appendix A.

\subsection{Horizon KSEs and lightcone integrability}

The gravitino and dilatino KSEs of IIB supergravity [19, 20] are

$$
\begin{aligned}
\left(\nabla_{M}-\right. & \left.\frac{i}{2} Q_{M}+\frac{i}{48} F_{M N_{1} N_{2} N_{3} N_{4}} \Gamma^{N_{1} N_{2} N_{3} N_{4}}\right) \epsilon \\
& -\frac{1}{96}\left(\Gamma_{M}{ }^{N_{1} N_{2} N_{3}} G_{N_{1} N_{2} N_{3}}-9 G_{M N_{1} N_{2}} \Gamma^{N_{1} N_{2}}\right) C * \epsilon=0 \\
& P_{M} \Gamma^{M} C * \epsilon+\frac{1}{24} G_{N_{1} N_{2} N_{3}} \Gamma^{N_{1} N_{2} N_{3}} \epsilon=0,
\end{aligned}
$$

respectively, where $Q$ is a $U(1)$ connection of $\lambda$.

Evaluating the gravitino KSE on the near horizon fields (2.1), one finds that these can be integrated along the lightcone directions. In particular setting $\epsilon=\epsilon_{+}+\epsilon_{-}, \Gamma_{ \pm} \epsilon_{ \pm}=0$, and solving the - component of the gravitino KSE, one finds that

$$
\epsilon_{+}=\phi_{+}, \quad \epsilon_{-}=\phi_{-}+r \Gamma_{-} \Theta_{+} \phi_{+},
$$

where $\phi_{ \pm}$do not depend on $r$, and the solution of the + component of the gravitino KSE gives

$$
\phi_{+}=\eta_{+}+u \Gamma_{+} \Theta_{-} \eta_{-}, \quad \phi_{-}=\eta_{-},
$$


where $\eta_{ \pm}$do not depend on both $u$ and $r$ coordinates, and where

$$
\Theta_{ \pm}=\left(\frac{1}{4} h_{i} \Gamma^{i} \pm \frac{i}{12} Y_{n_{1} n_{2} n_{3}} \Gamma^{n_{1} n_{2} n_{3}}\right)+\left(\frac{1}{96} H_{\ell_{1} \ell_{2} \ell_{3}} \Gamma^{\ell_{1} \ell_{2} \ell_{3}} \pm \frac{3}{16} \Phi_{i} \Gamma^{i}\right) C *
$$

Furthermore, the integrability of the gravitino KSE along the lightcone directions requires the following algebraic conditions

$$
\begin{gathered}
\left(\frac{1}{2} \Delta-\frac{1}{8} d h_{i j} \Gamma^{i j}\right) \phi_{+}+\frac{1}{16} L_{i j} \Gamma^{i j} C * \phi_{+}+2 \Theta_{-} \tau_{+}=0 \\
\left(\Delta h_{i}-\partial_{i} \Delta\right) \Gamma^{i} \phi_{+}+\left(-\frac{1}{2} d h_{i j} \Gamma^{i j}+\frac{i}{12} X_{\ell_{1} \ell_{2} \ell_{3} \ell_{4}} \Gamma^{\ell_{1} \ell_{2} \ell_{3} \ell_{4}}\right) \tau_{+}+\frac{1}{2} L_{i j} \Gamma^{i j} C * \tau_{+}=0,
\end{gathered}
$$

and

$$
\left(-\frac{1}{2} \Delta-\frac{1}{8} d h_{i j} \Gamma^{i j}+\frac{i}{48} X_{\ell_{1} \ell_{2} \ell_{3} \ell_{4}} \Gamma^{\ell_{1} \ell_{2} \ell_{3} \ell_{4}}\right) \eta_{-}+\frac{1}{8} L_{i j} \Gamma^{i j} C * \eta_{-}-2 \Theta_{+} \Theta_{-} \eta_{-}=0,
$$

where we have set

$$
\tau_{+}=\Theta_{+} \phi_{+}
$$

Next, we substitute the Killing spinor (2.5) into the gravitino KSE and evaluate the resulting expression along the directions transverse to the light cone to find

$$
\begin{gathered}
\tilde{\nabla}_{i} \phi_{+}+\left(-\frac{i}{2} \Lambda_{i}-\frac{1}{4} h_{i}-\frac{i}{4} Y_{i \ell_{1} \ell_{2}} \Gamma^{\ell_{1} \ell_{2}}+\frac{i}{12} \Gamma_{i}^{\ell_{1} \ell_{2} \ell_{3}} Y_{\ell_{1} \ell_{2} \ell_{3}}\right) \phi_{+} \\
+\left(\frac{1}{16} \Gamma_{i}^{j} \Phi_{j}-\frac{3}{16} \Phi_{i}-\frac{1}{96} \Gamma_{i}^{\ell_{1} \ell_{2} \ell_{3}} H_{\ell_{1} \ell_{2} \ell_{3}}+\frac{3}{32} H_{i \ell_{1} \ell_{2}} \Gamma^{\ell_{1} \ell_{2}}\right) C * \phi_{+}=0 \\
\tilde{\nabla}_{i} \tau_{+}+\left(-\frac{i}{2} \Lambda_{i}-\frac{3}{4} h_{i}+\frac{i}{4} Y_{i \ell_{1} \ell_{2}} \Gamma^{\ell_{1} \ell_{2}}-\frac{i}{12} \Gamma_{i}^{\ell_{1} \ell_{2} \ell_{3}} Y_{\ell_{1} \ell_{2} \ell_{3}}\right) \tau_{+} \\
+\left(-\frac{1}{16} \Gamma_{i}^{j} \Phi_{j}+\frac{3}{16} \Phi_{i}-\frac{1}{96} \Gamma_{i}^{\ell_{1} \ell_{2} \ell_{3}} H_{\ell_{1} \ell_{2} \ell_{3}}+\frac{3}{32} H_{i \ell_{1} \ell_{2}} \Gamma^{\ell_{1} \ell_{2}}\right) C * \tau_{+} \\
+\left(-\frac{1}{4} d h_{i j} \Gamma^{j}-\frac{i}{12} X_{i \ell_{1} \ell_{2} \ell_{3}} \Gamma^{\ell_{1} \ell_{2} \ell_{3}}\right) \phi_{+}+\left(\frac{1}{32} \Gamma_{i}^{\ell_{1} \ell_{2}} L_{\ell_{1} \ell_{2}}-\frac{3}{16} L_{i j} \Gamma^{j}\right) C * \phi_{+}=0
\end{gathered}
$$

and

$$
\begin{aligned}
& \tilde{\nabla}_{i} \eta_{-}+\left(-\frac{i}{2} \Lambda_{i}+\frac{1}{4} h_{i}+\frac{i}{4} Y_{i \ell_{1} \ell_{2}} \Gamma^{\ell_{1} \ell_{2}}-\frac{i}{12} \Gamma_{i}^{\ell_{1} \ell_{2} \ell_{3}} Y_{\ell_{1} \ell_{2} \ell_{3}}\right) \eta_{-}
\end{aligned}
$$

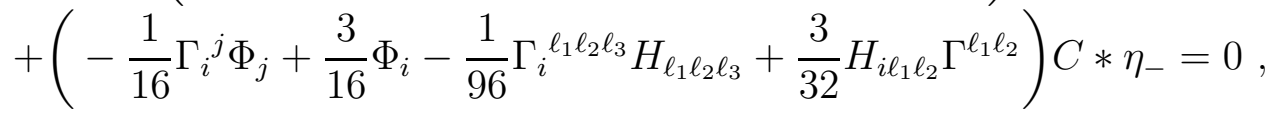

where $\Lambda$ is the restriction of $Q$ along $\mathcal{S}$. Note that $\Lambda$ is independent of $r, u$ because the near horizon scalars do not depend on these coordinates. 
It remains to evaluate the dilatino KSE (2.4) on the spinor (2.5). A direct substitution reveals that

$$
\begin{gathered}
\left(-\frac{1}{4} \Phi_{i} \Gamma^{i}+\frac{1}{24} H_{\ell_{1} \ell_{2} \ell_{3}} \Gamma^{\ell_{1} \ell_{2} \ell_{3}}\right) \phi_{+}+\xi_{i} \Gamma^{i} C * \phi_{+}=0, \\
\left(-\frac{1}{4} \Phi_{i} \Gamma^{i}-\frac{1}{24} H_{\ell_{1} \ell_{2} \ell_{3}} \Gamma^{\ell_{1} \ell_{2} \ell_{3}}\right) \tau_{+}-\xi_{i} \Gamma^{i} C * \tau_{+}+\frac{1}{8} L_{i j} \Gamma^{i j} \phi_{+}=0,
\end{gathered}
$$

and

$$
\left(\frac{1}{4} \Phi_{i} \Gamma^{i}+\frac{1}{24} H_{\ell_{1} \ell_{2} \ell_{3}} \Gamma^{\ell_{1} \ell_{2} \ell_{3}}\right) \eta_{-}+\xi_{i} \Gamma^{i} C * \eta_{-}=0 .
$$

This concludes the evaluation of the KSEs on the IIB near horizon geometries and their integration along the lightcone directions.

\subsection{Independent horizon KSEs}

The conditions on the Killing spinors we have found in the previous section are not independent. It is customary in the investigation of supersymmetric solutions that one first solves the KSEs. Then one imposes those Bianchi identities and field equations that are not implied as integrability conditions of the KSEs. Here, as in [8] and [9], we shall adopt a different strategy. We shall use the field equations and Bianchi identities to find which of the conditions implied by the KSEs presented in the previous section are independent.

To determine the independent KSEs on the horizon section $\mathcal{S}$ is more involved than similar results for M-horizons [9]. Because of this, we shall state here the result and the proof can be found in appendix B. In particular upon the use of the field equations and Bianchi identities of IIB supergravity, the independent KSEs are

$$
\nabla_{i}^{( \pm)} \eta_{ \pm} \equiv \tilde{\nabla}_{i} \eta_{ \pm}+\Psi_{i}^{( \pm)} \eta_{ \pm}=0
$$

and

$$
\mathcal{A}^{( \pm)} \eta_{ \pm}=0
$$

where

$$
\begin{aligned}
\Psi_{i}^{( \pm)}= & -\frac{i}{2} \Lambda_{i} \mp \frac{1}{4} h_{i} \mp \frac{i}{4} Y_{i \ell_{1} \ell_{2}} \Gamma^{\ell_{1} \ell_{2}} \mp \frac{i}{12} \Gamma_{i}^{{ }^{\ell} \ell_{2} \ell_{3}} Y_{\ell_{1} \ell_{2} \ell_{3}} \\
& +\left( \pm \frac{1}{16} \Gamma_{i}^{j} \Phi_{j} \mp \frac{3}{16} \Phi_{i}-\frac{1}{96} \Gamma^{{ }^{\ell} \ell_{2} \ell_{2} \ell_{3}} H_{\ell_{1} \ell_{2} \ell_{3}}+\frac{3}{32} H_{i \ell_{1} \ell_{2}} \Gamma^{\ell_{1} \ell_{2}}\right) C *
\end{aligned}
$$

and

$$
\mathcal{A}^{( \pm)}=\mp \frac{1}{4} \Phi_{i} \Gamma^{i}+\frac{1}{24} H_{\ell_{1} \ell_{2} \ell_{3}} \Gamma^{\ell_{1} \ell_{2} \ell_{3}}+\xi_{i} \Gamma^{i} C * .
$$


Furthermore if $\eta_{-}$solves (2.18) and (2.19), then

$$
\eta_{+}^{\prime}=\Gamma_{+} \Theta_{-} \eta_{-}
$$

also solves (2.18) and (2.19).

Therefore the independent KSEs are those that one finds after a naive restriction of the KSEs of IIB supergravity on $\mathcal{S}$ and after considering the lightcone projections of the Killing spinor. However, the additional property that $\eta_{+}^{\prime}$ solves the KSEs does not arise in this way and a more thorough analysis of the near horizon KSEs is required to establish this.

\section{Horizon Dirac equations}

We have seen that the gravitino KSE gives rise to two parallel transport equations on $\mathcal{S}$ associated with the covariant derivatives $\nabla^{( \pm)}(2.18)$. If $S^{ \pm}$are the complex chiral spin bundles over $\mathcal{S}$, then $\nabla^{ \pm}: \Gamma\left(S^{ \pm} \otimes \lambda^{\frac{1}{2}}\right) \rightarrow \Gamma\left(S^{ \pm} \otimes \lambda^{\frac{1}{2}}\right)$, where $\Gamma\left(S^{ \pm} \otimes \lambda^{\frac{1}{2}}\right)$ are the smooth sections of $S^{ \pm} \otimes \lambda^{\frac{1}{2}}$. In turn, one can define the associated Dirac operators

$$
\mathcal{D}^{( \pm)} \equiv \Gamma^{i} \nabla_{i}^{( \pm)}=\Gamma^{i} \tilde{\nabla}_{i}+\Psi^{ \pm}
$$

where

$$
\Psi^{ \pm} \equiv \Gamma^{i} \Psi_{i}^{( \pm)}=-\frac{i}{2} \Lambda_{i} \Gamma^{i} \mp \frac{1}{4} h_{i} \Gamma^{i} \pm \frac{i}{6} Y_{\ell_{1} \ell_{2} \ell_{3}} \Gamma^{\ell_{1} \ell_{2} \ell_{3}}+\left( \pm \frac{1}{4} \Phi_{i} \Gamma^{i}+\frac{1}{24} H_{\ell_{1} \ell_{2} \ell_{3}} \Gamma^{\ell_{1} \ell_{2} \ell_{3}}\right) C *(3
$$

Clearly the $\nabla^{ \pm}$parallel spinors are zero modes of $\mathcal{D}^{( \pm)}$. Here we shall prove the converse. In particular, we shall show that all zero modes of the horizon Dirac equations $\mathcal{D}^{( \pm)}$are Killing spinors, i.e. they are parallel with respect to the $\nabla^{ \pm}$connections and $\mathcal{A}^{( \pm)} \eta_{ \pm}=0$. Therefore, we shall establish

$$
\nabla^{( \pm)} \eta_{ \pm}=0, \quad \mathcal{A}^{( \pm)} \eta_{ \pm}=0 \Longleftrightarrow \mathcal{D}^{( \pm)} \eta_{ \pm}=0
$$

In addition, we shall demonstrate that

$$
\left\|\eta_{+}\right\|=\text {const. }
$$

We shall prove the above statements separately for the $\mathcal{D}^{( \pm)}$horizon operators.

\subsection{A maximum principle for $\left\|\eta_{+}\right\|^{2}$}

As we have mentioned, if $\eta_{+}$is Killing spinor then $\eta_{+}$is a zero mode of $\mathcal{D}^{(+)}$which demonstrates (3.3) in one direction. It remains to show the opposite direction of (3.3), and (3.4). For this, we shall formulate a maximal principle for the scalar function $\left\|\eta_{+}\right\|^{2}$ of $\mathcal{S}$. In particular, assuming that $\mathcal{D}^{(+)} \eta_{+}=0$ and after some extensive Clifford algebra which is described in detail in appendix $\mathrm{C}$, one establishes

$$
\tilde{\nabla}^{i} \tilde{\nabla}_{i}\left\|\eta_{+}\right\|^{2}-h^{i} \tilde{\nabla}_{i}\left\|\eta_{+}\right\|^{2}=2\left\|\nabla^{(+)} \eta_{+}\right\|^{2}+\left\|\mathcal{A}^{(+)} \eta_{+}\right\|^{2} .
$$


Since $\mathcal{S}$ is compact and the right hand side of (3.5) is positive, an application of the maximum principle implies that (3.4) holds and that $\eta_{+}$is a Killing spinor. This establishes both (3.4) and the opposite direction in (3.3) for the $\eta_{+}$spinors.

We remark that $\left\|\eta_{+}\right\|=$const implies the following conditions

$$
-\Delta\left\|\eta_{+}\right\|^{2}+4\left\|\Theta_{+} \eta_{+}\right\|^{2}=0, \quad \operatorname{Re}\left\langle\eta_{+}, \Gamma_{i} \Theta_{+} \eta_{+}\right\rangle=0
$$

Alternatively, these conditions can also be derived from the requirement that the vector bilinear in section 6 is Killing.

\subsection{A Lichnerowicz theorem for $\mathcal{D}^{(-)}$}

Again if $\eta_{-}$is a Killing spinor, then $\eta_{-}$is a zero mode of the horizon Dirac operator $\mathcal{D}^{(-)}$ which establishes one direction in (3.3). To prove the converse, we use

$$
\Gamma^{i j} \tilde{\nabla}_{i} \tilde{\nabla}_{j} \eta_{-}=-\frac{1}{4} \tilde{R} \eta_{-},
$$

to find after some extensive Clifford algebra, which is described in appendix C, the identity

$$
\int_{\mathcal{S}}\left\|\mathcal{D}^{(-)} \eta_{-}\right\|^{2}=\int_{\mathcal{S}}\left\|\nabla^{(-)} \eta_{-}\right\|^{2}+\frac{1}{2} \int_{\mathcal{S}}\left\|\mathcal{A}^{(-)} \eta_{-}\right\|^{2}+\int_{\mathcal{S}} \operatorname{Re}\left\langle\mathcal{B} \eta_{-}, \mathcal{D}^{(-)} \eta_{-}\right\rangle+\cdots
$$

where $\mathcal{B}$ is a Clifford algebra element that depends on the fluxes, and the dots represent surface terms and terms that depend on the field equations and Bianchi identities, see appendix $\mathrm{C}$. Clearly the surface terms vanish because $\mathcal{S}$ is compact without boundary. It is evident from (3.8) that if $\mathcal{D}^{(-)} \eta_{-}=0$ and the field equations and Bianchi identities of IIB supergravity are satisfied, then $\eta_{-}$is a Killing spinor. This establishes the opposite direction in (3.3) for the $\eta_{-}$spinors.

\section{Index theorem and supersymmetry}

We shall now establish the relation (1.1) between the number of supersymmetries $N$ preserved by the IIB horizons and the index of Dirac operator presented in the introduction. First observe that

$$
N=N_{+}+N_{-},
$$

where

$$
N_{ \pm}=\operatorname{dim} \operatorname{Ker}\left(\nabla^{( \pm)}, \mathcal{A}^{( \pm)}\right) .
$$

On the other hand, the Lichnerowicz type theorem established in (3.3) implies that

$$
N_{ \pm}=\operatorname{dim} \operatorname{Ker}\left(\mathcal{D}^{( \pm)}\right)
$$

Next, to apply the index theorem, first observe that the spinor bundle over the spacetime twisted by $\lambda^{\frac{1}{2}}$ when restricted on $\mathcal{S}$ decomposes as $S \otimes \lambda^{\frac{1}{2}}=S_{+} \otimes \lambda^{\frac{1}{2}} \oplus S_{-} \otimes \lambda^{\frac{1}{2}}$. In addition since the IIB spinors lie in the positive chirality Weyl representation of $\operatorname{Spin}_{c}(9,1)$, 
$S_{+} \otimes \lambda^{\frac{1}{2}}$ can be identified with the positive chirality spinor bundle $\tilde{S}_{+} \otimes \lambda^{\frac{1}{2}}$ of $\operatorname{Spin}_{c}(8)$. On the other hand $S_{-} \otimes \lambda^{\frac{1}{2}}$ can be identified with the negative chirality spinor bundle $\tilde{S}_{-} \otimes \lambda^{\frac{1}{2}}$ of $\operatorname{Spin}_{c}(8)$ because if $\alpha_{-}$is a section ${ }^{5}$ of $\tilde{S}_{-} \otimes \lambda^{\frac{1}{2}}$, then $\eta_{-}=\Gamma_{-} \alpha_{-}$is a section of $S_{-} \otimes \lambda^{\frac{1}{2}}$ and vice versa. Using these identifications of spinor bundles, one concludes that $\mathcal{D}^{(+)}: \Gamma\left(\tilde{S}_{+} \otimes \lambda^{\frac{1}{2}}\right) \rightarrow \Gamma\left(\tilde{S}_{-} \otimes \lambda^{\frac{1}{2}}\right)$, where $\Gamma\left(\tilde{S}_{ \pm} \otimes \lambda^{\frac{1}{2}}\right)$ denotes the smooth section of these bundles. The horizon Dirac operator $\mathcal{D}^{(+)}$has the same principal symbol as a $\lambda^{\frac{1}{2}}$ twisted Dirac operator $\mathcal{D}_{\lambda}$ and therefore the same index. As a result

$$
\operatorname{Index}\left(\mathcal{D}^{(+)}\right)=\operatorname{dim} \operatorname{Ker}\left(\mathcal{D}^{(+)}\right)-\operatorname{dim} \operatorname{Ker}\left(\left(\mathcal{D}^{(+)}\right)^{\dagger}\right)=2 \operatorname{Index}\left(\mathcal{D}_{\lambda}\right) .
$$

The factor of 2 appears in the right hand side of (4.4) because we count the dimension of the index of $\mathcal{D}^{(+)}$over the real numbers.

To proceed, we shall show that

$$
N_{-}=\operatorname{dim} \operatorname{Ker}\left(\left(\mathcal{D}^{(+)}\right)^{\dagger}\right)
$$

For this observe that

$$
\left(\mathcal{D}^{(+)}\right)^{\dagger}=-\Gamma^{i} \tilde{\nabla}_{i}+\frac{i}{2} \Lambda_{i} \Gamma^{i}-\frac{1}{4} h_{i} \Gamma^{i}+\frac{i}{6} Y_{\ell_{1} \ell_{2} \ell_{3}} \Gamma^{\ell_{1} \ell_{2} \ell_{3}}+\left(\frac{1}{4} \Phi_{i} \Gamma^{i}-\frac{1}{24} H_{\ell_{1} \ell_{2} \ell_{3}} \Gamma^{\ell_{1} \ell_{2} \ell_{3}}\right) C *
$$

where the conjugate is taken with the real part of the hermitian inner product in $\tilde{S}_{-} \otimes \lambda^{\frac{1}{2}}$, and that

$$
\mathcal{D}^{(-)} \Gamma_{-}=\Gamma_{-}\left(\mathcal{D}^{(+)}\right)^{\dagger}
$$

Therefore the dimension of the kernel of $\mathcal{D}^{(-)}$is the same as that of $\left(\mathcal{D}^{(+)}\right)^{\dagger}$ which establishes (4.5). Next combining (4.1), (4.4) and (4.5), one can show (1.1) in the introduction with $N_{-}$given by $(4.2)$.

\section{$5 \quad \eta_{+}$from $\eta_{-}$Killing spinors}

In this section, we shall explore further the observation made in section 2.3 that if $\eta_{-} \neq 0$ is a Killing spinor, then $\eta_{+}^{\prime}=\Gamma_{+} \Theta_{-} \eta_{-}$is also a Killing spinor. In particular, we shall show that

$$
\operatorname{Ker} \Theta_{-}=\{0\}
$$

for all horizons with fluxes, otherwise the metric decomposes as a product $\mathbb{R}^{1,1} \times X^{8}$, the holonomy of $X^{8}$ is a subgroup of $\operatorname{Spin}(7)$ and all the fluxes vanish. Therefore for horizons with non-trivial fluxes, $\Gamma_{+} \Theta_{-}$is an injection and so $\operatorname{Index}\left(D_{\lambda}\right) \geq 0$.

To show this suppose that there is $\eta_{-} \neq 0$ Killing spinor, i.e. $\nabla^{(-)} \eta_{-}=\mathcal{A}^{(-)} \eta_{-}=0$, such that

$$
\Theta_{-} \eta_{-}=0
$$

\footnotetext{
${ }^{5}$ Note that the subscript in $\alpha_{-}$denotes $\operatorname{Spin}(8)$ chirality while the subscript in $\eta_{-}$denotes a $\Gamma_{-}$ projection.
} 
To proceed, note that (2.10) together with (5.2) imply that

$$
\left(-\frac{1}{2} \Delta-\frac{1}{8} d h_{i j} \Gamma^{i j}+\frac{i}{48} X_{\ell_{1} \ell_{2} \ell_{3} \ell_{4}} \Gamma^{\ell_{1} \ell_{2} \ell_{3} \ell_{4}}\right) \eta_{-}+\frac{1}{8} L_{i j} \Gamma^{i j} C * \eta_{-}=0 .
$$

In turn this implies that

$$
\Delta\left\langle\eta_{-}, \eta_{-}\right\rangle=0,
$$

where we have used the identity $\left\langle\eta_{-}, \Gamma_{i j} C * \eta_{-}\right\rangle=0$. Since $\eta_{-}$is nowhere vanishing,

$$
\Delta=0 .
$$

Next, using (2.14), we compute

$$
\begin{aligned}
\tilde{\nabla}_{i}\left\langle\eta_{-}, \eta_{-}\right\rangle & =-\frac{1}{2} h_{i}\left\langle\eta_{-}, \eta_{-}\right\rangle+\left\langle\eta_{-},-\frac{i}{2} Y_{i \ell_{1} \ell_{2}} \Gamma^{\ell_{1} \ell_{2}} \eta_{-}\right\rangle \\
& +2 \operatorname{Re}\left(\left\langle\eta_{-}, \Gamma_{i}\left(-\frac{3}{16} \Phi_{j} \Gamma^{j}+\frac{1}{96} H_{\ell_{1} \ell_{2} \ell_{3}} \Gamma^{\ell_{1} \ell_{2} \ell_{3}}\right) C * \eta_{-}\right\rangle .\right.
\end{aligned}
$$

Then, on substituting (5.2) into (5.6) in order to eliminate the $\Phi$ and $H$-terms, one obtains

$$
\tilde{\nabla}_{i}\left\langle\eta_{-}, \eta_{-}\right\rangle=-h_{i}\left\langle\eta_{-}, \eta_{-}\right\rangle .
$$

Then on using (A.7), together with $\Delta=0,(5.7)$ implies that

$$
\tilde{\nabla}^{i} \tilde{\nabla}_{i}\left\langle\eta_{-}, \eta_{-}\right\rangle=\left(\frac{4}{3} Y_{\ell_{1} \ell_{2} \ell_{3}} Y^{\ell_{1} \ell_{2} \ell_{3}}+\frac{3}{4} \Phi_{i} \bar{\Phi}^{i}+\frac{1}{24} H_{\ell_{1} \ell_{2} \ell_{3}} \bar{H}^{\ell_{1} \ell_{2} \ell_{3}}\right) \cdot\left\langle\eta_{-}, \eta_{-}\right\rangle
$$

On integrating both sides of this expression over $\mathcal{S}$, one finds that

$$
Y=0, \quad H=0, \quad \Phi=0 .
$$

Returning to (A.7), we have

$$
\tilde{\nabla}^{i} h_{i}=h^{2}
$$

and so integrating both sides of this expression over $\mathcal{S}$ one finds

$$
h=0
$$

as well. With these conditions, it is straightforward to see that the Bianchi identities also imply that

$$
X=0, \quad L=0 .
$$

Hence, the 5-form $F$ and complex 3-form $G$ vanish.

It remains to consider the complex 1 -form $\xi$, and the connection $\Lambda$. The spacetime is $\mathbb{R}^{1,1} \times \mathcal{S}$, where $\mathcal{S}$ admits a spinor $\eta_{\text {- satisfying }}$

$$
\tilde{\nabla}_{i} \eta_{-}=\frac{i}{2} \Lambda_{i} \eta_{-},
$$


whose Ricci tensor is given as

$$
\tilde{R}_{i j}=2 \xi_{(i} \bar{\xi}_{j)},
$$

and the algebraic KSE (2.17) reduces to

$$
\bar{\xi}_{i} \Gamma^{i} \eta_{-}=0 .
$$

We also have

$$
\tilde{\nabla}^{i} \xi_{i}-2 i \Lambda^{i} \xi_{i}=0
$$

and

$$
d \Lambda=-i \xi \wedge \bar{\xi} .
$$

Note that (5.13) implies that $\mathcal{S}$ is $\mathrm{Kähler}^{6}$. There are two cases to consider, corresponding as to whether $\eta_{-}$is a pure spinor, or not.

First, observe that (5.15) implies that either $\eta_{-}$is a pure spinor or $\xi=0$. If $\xi=0, \mathcal{S}$ is Ricci flat and $d \Lambda=0$. Thus $\mathcal{S}$ up to a finite cover has holonomy contained in $\operatorname{Spin}(7)$.

Next, suppose that $\eta_{-}$is pure. Then (5.15) implies that

$$
\xi^{i} \xi_{i}=0 .
$$

Using this, the bosonic field equations and Bianchi identities, together with $\xi^{i} \xi_{i}=0$, one can show that

$$
\tilde{\nabla}_{i} \tilde{\nabla}^{i}\left(\xi^{j} \bar{\xi}_{j}\right)=2\left(\tilde{\nabla}_{(i} \xi_{j)}-2 i \Lambda_{(i} \xi_{j)}\right)\left(\tilde{\nabla}^{(i} \bar{\xi}^{j)}+2 i \Lambda^{(i} \bar{\xi}^{j)}\right)+6 \xi^{i} \bar{\xi}_{i} \xi^{j} \bar{\xi}_{j} .
$$

On applying the maximum principle, one finds ${ }^{7}$

$$
\xi=0 .
$$

Therefore all the fluxes vanish and establishes our result.

\section{The dynamical $\mathfrak{s l}(2, \mathbb{R})$ symmetry of IIB horizons}

\subsection{Killing vectors}

IIB horizons with $N_{-}=0$ coincide with those for which the bi-linear matching condition has been imposed and their geometry has already been investigated in [18]. Here we shall explore some aspects of the geometry of horizons with $N_{-} \neq 0$. In particular, we

\footnotetext{
${ }^{6}$ This can be shown by observing that 2 -form spinor bilinear constructed from $\eta_{-}$is not degenerate and it is covariantly constant with respect to the Levi-Civita connection.

${ }^{7}$ The application of the maximum principle requires that $\xi$ is at least $C^{2}$ differentiable. So our results imply that the cosmic string solutions of [21] and the D7 branes of [22] with compact transverse space, including those with 24 strings and 24 D7-branes, respectively, cannot be more than $C^{1}$ differentiable.
} 
shall demonstrate that if $N_{-} \neq 0$, then the IIB horizons with non-trivial fluxes admit an $\mathfrak{s l}(2, \mathbb{R})$ symmetry subalgebra. First note that the IIB horizons (2.1) are invariant under the symmetries generated by the vector fields $\partial_{u}$ and $u \partial_{u}-r \partial_{r}$. Here we shall show that they admit an additional symmetry which enhances the symmetry algebra to $\mathfrak{s l}(2, \mathbb{R})$. Such an additional symmetry is a consequence of the supersymmetry enhancement that we have already demonstrated for IIB horizons with non-trivial fluxes and $N_{-} \neq 0$. Both the additional supersymmetry and $\mathfrak{s l}(2, \mathbb{R})$ symmetry are dynamical as they arise as a consequence of the IIB field equations.

Using (2.5) and (2.6), one finds that the most general Killing spinor is

$$
\epsilon=\eta_{+}+u \Gamma_{+} \Theta_{-} \eta_{-}+\eta_{-}+r \Gamma_{-} \Theta_{+} \eta_{+}+r u \Gamma_{-} \Theta_{+} \Gamma_{+} \Theta_{-} \eta_{-} \cdot
$$

Since we have assumed that $N_{-} \neq 0$, there is an $\eta_{-} \neq 0$ which solves the KSEs $\nabla^{(-)} \eta_{-}=$ $\mathcal{A}^{(-)} \eta_{-}=0$ on the horizon section $\mathcal{S}$. Furthermore, since we have also assumed that the horizon does not have trivial fluxes $\Theta_{-}$is an injection and so there is a spinor $\eta_{+}=$ $\Gamma_{+} \Theta_{-} \eta_{-}$which also solves the KSEs on the horizon section $\mathcal{S}$, i.e. $\nabla^{(+)} \eta_{+}=\mathcal{A}^{(+)} \eta_{+}=0$. Since $\eta_{-}$and $\eta_{+}$are linearly independent, they give rise to two Killing spinors (6.21) which can be constructed from the pairs $\left(\eta_{-}, 0\right)$ and $\left(\eta_{-}, \eta_{+}\right)$. After a rearrangement, the two Killing spinors (6.21) can be written as

$$
\epsilon_{1}=\eta_{-}+u \eta_{+}+r u \Gamma_{-} \Theta_{+} \eta_{+}, \quad \epsilon_{2}=\eta_{+}+r \Gamma_{-} \Theta_{+} \eta_{+}, \quad \eta_{+}=\Gamma_{+} \Theta_{-} \eta_{-} .
$$

To continue, we shall use the property of the KSEs of IIB supergravity that if $\zeta_{1}$ and $\zeta_{2}$ are Killing spinors, then the 1-form bilinear ${ }^{8}$

$$
K=\operatorname{Re}\left\langle\left(\Gamma_{+}-\Gamma_{-}\right) \zeta_{1}, \Gamma_{A} \zeta_{2}\right\rangle e^{A}
$$

is associated with a Killing vector which also preserves the 1-, 3- and 5-form fluxes [23, 28], see appendix D for a short proof of this statement. In particular, from the two Killing spinors (6.22), one can construct three 1-form bi-linears. A substitution of (6.22) into (6.23) reveals

$$
\begin{aligned}
K_{1}=\operatorname{Re}\left\langle\left(\Gamma_{+}-\Gamma_{-}\right) \epsilon_{1}, \Gamma_{A} \epsilon_{2}\right\rangle e^{A} & =\left(2 r \operatorname{Re}\left\langle\Gamma_{+} \eta_{-}, \Theta_{+} \eta_{+}\right\rangle+u r^{2} \Delta\left\|\eta_{+}\right\|^{2}\right) \mathbf{e}^{+} \\
& -2 u\left\|\eta_{+}\right\|^{2} \mathbf{e}^{-}+V_{i} \mathbf{e}^{i}, \\
K_{2}=\operatorname{Re}\left\langle\left(\Gamma_{+}-\Gamma_{-}\right) \epsilon_{2}, \Gamma_{A} \epsilon_{2}\right\rangle e^{A} & =r^{2} \Delta\left\|\eta_{+}\right\|^{2} \mathbf{e}^{+}-2\left\|\eta_{+}\right\|^{2} \mathbf{e}^{-} \\
K_{3}=\operatorname{Re}\left\langle\left(\Gamma_{+}-\Gamma_{-}\right) \epsilon_{1}, \Gamma_{A} \epsilon_{1}\right\rangle e^{A} & =\left(2\left\|\eta_{-}\right\|^{2}+4 r u \operatorname{Re}\left\langle\Gamma_{+} \eta_{-}, \Theta_{+} \eta_{+}\right\rangle+r^{2} u^{2} \Delta\left\|\eta_{+}\right\|^{2}\right) \mathbf{e}^{+} \\
& -2 u^{2}\left\|\eta_{+}\right\|^{2} \mathbf{e}^{-}+2 u V_{i} \mathbf{e}^{i},
\end{aligned}
$$

where we have set

$$
V_{i}=\operatorname{Re}\left\langle\Gamma_{+} \eta_{-}, \Gamma_{i} \eta_{+}\right\rangle
$$

\footnotetext{
${ }^{8}$ The 1 -form bilinear which gives rise to a Killing vector is that associated with the real part of the Dirac inner product of $\operatorname{Spin}(9,1)$, see [23].
} 
and we have used (3.6) to simplify the components of the $K_{a}, a=1,2,3$. By construction, all three 1-forms must give rise to symmetries of the IIB background. In particular, one has that

$$
\mathcal{L}_{K_{a}} g=0, \quad \mathcal{L}_{K_{a}} F=0, \quad \mathcal{L}_{K_{a}} P=2 i Q_{a} P, \quad \mathcal{L}_{K_{a}} G=i Q_{a} G, \quad \mathcal{L}_{K_{a}} d Q=0,
$$

where $Q_{a}=i_{K_{a}} Q$. Observe that the $P$ and $G$ fluxes are invariant up to a $U(1)$ transformation, and we shall assume that $d Q$ is equivariant under the group action. To find the conditions that this imposes on the geometry of $\mathcal{S}$, we shall investigate two different cases depending on whether $V=0$ or not.

\section{2 $V \neq 0$}

To find the restrictions on the geometry of $\mathcal{S}$ and the fluxes imposed by $K_{1}, K_{2}$ and $K_{3}$, we decompose the conditions (6.26) along the lightcone and transverse directions and after some straightforward computation, we find that

$$
\begin{array}{r}
\tilde{\nabla}_{(i} V_{j)}=0, \quad \tilde{\mathcal{L}}_{V} h=0, \quad \tilde{\mathcal{L}}_{V} \Delta=0, \quad \tilde{\mathcal{L}}_{V} Y=0, \\
\tilde{\mathcal{L}}_{V} L=i \Lambda_{V} L, \quad \tilde{\mathcal{L}}_{V} \Phi=i \Lambda_{V} \Phi, \quad \tilde{\mathcal{L}}_{V} H=i \Lambda_{V} H, \quad \tilde{\mathcal{L}}_{V} \xi=2 i \Lambda_{V} \xi, \quad \mathcal{L}_{V} d \Lambda=0
\end{array}
$$

where $\Lambda_{V}=i_{V} \Lambda$. Therefore, $\mathcal{S}$ admits an isometry generated by $V$ which leaves $h, \Delta, Y$, and transforms $L, \Phi, H$ and $\xi$ up to a $U(1)$ transformation. In addition, one finds the conditions

$$
\begin{aligned}
& -2\left\|\eta_{+}\right\|^{2}-h_{i} V^{i}+2 \operatorname{Re}\left\langle\Gamma_{+} \eta_{-}, \Theta_{+} \eta_{+}\right\rangle=0, \quad i_{V}(d h)+2 d \operatorname{Re}\left\langle\Gamma_{+} \eta_{-}, \Theta_{+} \eta_{+}\right\rangle=0 \\
& 2 \operatorname{Re}\left\langle\Gamma_{+} \eta_{-}, \Theta_{+} \eta_{+}\right\rangle-\Delta\left\|\eta_{-}\right\|^{2}=0, \quad V+\left\|\eta_{-}\right\|^{2} h+d\left\|\eta_{-}\right\|^{2}=0 .
\end{aligned}
$$

These follow from the KSEs and the field equations, and we shall use them later to further simplify the vector fields associated with $K_{1}, K_{2}$ and $K_{3}$. Notice that the last equality in (6.28) expresses $V$ in terms of $h$. A similar relation has been derived for heterotic and M horizons [9], [24]. Furthermore, one has that

$$
\mathcal{L}_{V}\left\|\eta_{-}\right\|^{2}=0 .
$$

It is likely that in addition to the Killing vectors associated with $K_{1}, K_{2}$ and $K_{3}$, there are additional conditions on the geometry of $\mathcal{S}$. We shall not elaborate on these here. The results will be reported elsewhere.

\subsection{1 $V=0$}

A special case arises whenever $V=0$. In this case, the group action generated by $K_{1}, K_{2}$ and $K_{3}$ has only 2-dimensional orbits. A direct substitution of this condition in (6.28) reveals that

$$
\Delta\left\|\eta_{-}\right\|^{2}=2\left\|\eta_{+}\right\|^{2}, \quad h=\Delta^{-1} d \Delta .
$$

Since $d h=0$, and $h$ is exact, such horizons are static. Using this, the field equation (A.11) implies that

$$
X=L=0
$$


After a coordinate transformation $r \rightarrow \Delta r$, the near horizon geometry becomes a warped product of $A d S_{2}$ with $\mathcal{S}, A d S_{2} \times{ }_{w} \mathcal{S}$. Therefore, in this way one can identify the most general $A d S_{2}$ backgrounds of IIB supergravity. As a consequence of our results, IIB $A d S_{2}$ backgrounds preserve at least 2 supersymmetries.

\section{3 $\mathfrak{s l}(2, \mathbb{R})$ symmetry of IIB horizons}

It remains to show that all IIB horizons with non-trivial fluxes and $N_{-} \neq 0$ admit an $\mathfrak{s l}(2, \mathbb{R})$ symmetry. For this, we use the various identities derived in the previous section (6.28) to write the vector fields associated to the 1 -forms $K_{1}, K_{2}$ and $K_{3}(6.24)$ as

$$
\begin{aligned}
& K_{1}=-2 u\left\|\eta_{+}\right\|^{2} \partial_{u}+2 r\left\|\eta_{+}\right\|^{2} \partial_{r}+V^{i} \tilde{\partial}_{i} \\
& K_{2}=-2\left\|\eta_{+}\right\|^{2} \partial_{u} \\
& K_{3}=-2 u^{2}\left\|\eta_{+}\right\|^{2} \partial_{u}+\left(2\left\|\eta_{-}\right\|^{2}+4 r u\left\|\eta_{+}\right\|^{2}\right) \partial_{r}+2 u V^{i} \tilde{\partial}_{i}
\end{aligned}
$$

where we have used the same symbol for the 1-forms and the associated vector fields. A direct computation then reveals using (6.29) that

$$
\left[K_{1}, K_{2}\right]=2\left\|\eta_{+}\right\|^{2} K_{2}, \quad\left[K_{2}, K_{3}\right]=-4\left\|\eta_{+}\right\|^{2} K_{1}, \quad\left[K_{3}, K_{1}\right]=2\left\|\eta_{+}\right\|^{2} K_{3} .
$$

Therefore all such IIB horizons with non-trivial fluxes admit an $\mathfrak{s l}(2, \mathbb{R})$ symmetry subalgebra. Note also that the orbits of the vector fields are either 2- or 3-dimensional depending on whether $V=0$ or not. As we have seen in the $V=0$ case, the orbits are $A d S_{2}$. Furthermore, if the fluxes are trivial, the spacetime is isometric to $\mathbb{R}^{2} \times \mathcal{S}$ and $\mathcal{S}$ admits at least one isometry. The symmetry group in this case has an $\mathfrak{s o}(1,1) \oplus \mathfrak{u}(1)$ subalgebra.

\section{Common sector horizons, and horizons with 5-form fluxes}

\section{$7.1 \quad 5$-form flux horizons}

IIB horizons with only 5-form fluxes have been investigated before in [25] using the bilinear matching condition which requires that $\eta_{-}=0$. Therefore the horizons that have been investigated so far are those with $N_{-}=0$, and it has been found that the near horizon sections include manifolds with a 2-SCYT structure. Since $N_{-}=0$, the formula (1.1) implies $N=2 \operatorname{Index}(D)$ and so the number of supersymmetries is a topological invariant. Note that for all these horizons the line bundle $\lambda$ associated with the IIB scalars is trivial.

Next we shall assume that $N_{-} \neq 0$. As we have already shown all such horizons with non-trivial fluxes admit an $\mathfrak{s l}(2, \mathbb{R})$ symmetry. Here we shall show that under an additional assumption on $V$ all such horizons have vanishing Dirac index and admit

$$
N=4 k,
$$

supersymmetries. 
To see this, we shall consider two separate cases. First take $V \neq 0$. Assuming that the isometries generated by $V$ can be integrated to a circle action on $\mathcal{S}$, one can show [26] that $\hat{A}[\mathcal{S}]=0$ and so the index of the Dirac operator vanishes. As a result we have that $N_{+}=N_{-}$. Since the KSEs of IIB supergravity for backgrounds with only 5 -form fluxes are linear over the complex numbers, $N_{-}$is even and so such horizons preserve $4 \mathrm{k}$ supersymmetries, $N_{-}=2 k$.

Next suppose that $V=0$. In this case, we have shown that the horizons with nontrivial fluxes are warped products $A d S_{2} \times_{w} \mathcal{S}$. Observe that (6.30) implies that $\Delta$ is no-where vanishing, and using $h=\Delta^{-1} d \Delta$, the Einstein equation of $\mathcal{S}$ implies that the metric

$$
g^{\prime}=\Delta^{-\frac{1}{7}} g
$$

has positive Ricci scalar, $\tilde{R}\left(g^{\prime}\right)>0$. As a result, the index of the Dirac operator vanishes and so again such horizons preserve $4 k$ supersymmetries. Clearly in this case, there is a topological obstruction for $\mathcal{S}$ to be a solution of the KSEs which is the vanishing of $\hat{A}$-genus.

The field equations impose additional conditions on $A d S_{2} \times{ }_{w} \mathcal{S}$ horizons. For example, they imply that $Y$ is a harmonic 3 -form on $\mathcal{S}$. The solution of the field equations and the geometry of $\mathcal{S}$ will be explored elsewhere.

\subsection{Common sector}

Another consistent truncation of IIB supergravity is to the common sector. The investigation of common sector horizons and their relation to the heterotic ones [24] have already been explored in [18] using the bilinear matching condition which sets $\eta_{-}=0$. Here we shall demonstrate that the bilinear matching condition does not impose a restriction on common sector horizons and the investigation of their geometry reduces to that of the heterotic horizons described in [24]. To see this, it is convenient to work in the string frame $^{9}$. It is well known that in this frame, the KSEs of IIB supergravity factorize to two pairs ${ }^{10}$ of KSEs depending on the string frame metric, the 3 -form field strength $\mathcal{H}$, $d \mathcal{H}=0$, and the dilaton $\phi$, as

$$
\begin{array}{ll}
\hat{\nabla} \hat{\epsilon}=0, & \hat{\mathcal{A}} \hat{\epsilon}=0, \\
\check{\nabla} \check{\epsilon}=0, & \mathcal{\mathcal { A }} \check{\epsilon}=0,
\end{array}
$$

where $\hat{\nabla}=\nabla+\frac{1}{2} \mathcal{H}$ and $\hat{\mathcal{A}}=\Gamma^{M} \partial_{M} \phi-\frac{1}{12} \mathcal{H}_{M N R} \Gamma^{M N R}$, and $\check{\nabla}$ and $\check{\mathcal{A}}$ can be derived from these after setting $\mathcal{H}$ to $-\mathcal{H}$.

To continue, we can apply our results to one of two pairs of KSEs above. In fact it is instructive to repeat the calculation we have done for IIB for the common sector in the string frame. The common sector horizon metric is as in (2.1) and using $d \mathcal{H}=0$, one can write

$$
\mathcal{H}=\mathbf{e}^{+} \wedge \mathbf{e}^{-} \wedge S+r \mathbf{e}^{+} \wedge d_{h} S+\tilde{\mathcal{H}}, \quad d \tilde{\mathcal{H}}=0,
$$

\footnotetext{
${ }^{9}$ The relation of the IIB horizon fields to the string frame common sector horizon fields can be found in [18]. From here on all the fields are those of the common sector.

${ }^{10}$ We have used the signs \pm in [18] to denote these two pairs of KSEs. We have changed the notation here to avoid confusion with the labeling of lightcone projections.
} 
where $S$ and $\tilde{\mathcal{H}}$ are a 1 -form and a 3 -form on $\mathcal{S}$, respectively. Without loss of generality consider the first pair of KSEs in (7.3). Solving along the light cone directions, one finds

$$
\hat{\epsilon}_{+}=\hat{\phi}_{+}, \quad \hat{\epsilon}_{-}=\hat{\phi}_{-}+r \Gamma_{-} \Theta_{+} \hat{\phi}_{+},
$$

where $\hat{\phi}_{ \pm}$do not depend on $r$, and

$$
\hat{\phi}_{+}=\hat{\eta}_{+}+u \Gamma_{+} \Theta_{-} \hat{\eta}_{-}, \quad \hat{\phi}_{-}=\hat{\eta}_{-},
$$

where $\hat{\eta}_{ \pm}$do not depend on both $r$ and $u$, and

$$
\Theta_{ \pm}=\frac{1}{4} h_{i} \Gamma^{i} \mp \frac{1}{4} S_{i} \Gamma^{i}
$$

An application of our IIB results implies that the remaining independent KSEs are

$$
\begin{aligned}
\hat{\tilde{\nabla}}_{i} \hat{\eta}_{ \pm} \mp \frac{1}{4} h_{i} \hat{\eta}_{ \pm} \pm \frac{1}{4} S_{i} \hat{\eta}_{ \pm} & =0 \\
\tilde{\partial}_{i} \phi \Gamma^{i} \hat{\eta}_{ \pm} \pm \frac{1}{2} S_{i} \Gamma^{i} \hat{\eta}_{ \pm}-\frac{1}{12} \tilde{\mathcal{H}}_{i j k} \Gamma^{i j k} \hat{\eta}_{ \pm} & =0 .
\end{aligned}
$$

The analysis can proceed as in the general IIB case. In particular, for horizons with non-trivial fluxes, one has $\operatorname{Ker} \Theta_{-}=\{0\}$. To establish this, we make use of the following common sector field equations:

$$
\begin{gathered}
\tilde{\nabla}^{i} h_{i}=2 \Delta+h^{2}-S^{2}+2 h^{i} \tilde{\nabla}_{i} \phi, \\
\tilde{\nabla}^{i} S_{i}-2 S^{i} \tilde{\nabla}_{i} \phi=0,
\end{gathered}
$$

and

$$
\tilde{\nabla}^{i} \tilde{\nabla}_{i} \phi=h^{i} \tilde{\nabla}_{i} \phi+2 \tilde{\nabla}^{i} \phi \tilde{\nabla}_{i} \phi+\frac{1}{2} S^{2}-\frac{1}{12} \tilde{\mathcal{H}}_{i j k} \tilde{\mathcal{H}}^{i j k}
$$

These conditions are obtained from the +- components of the Einstein, 3-form gauge, and the dilaton field equations.

Then, if $\operatorname{Ker} \Theta_{-} \neq\{0\}$, one obtains

$$
S=-h
$$

and furthermore,

$$
\Delta=0
$$

as a consequence of one of the algebraic conditions obtained from the KSE. In addition, the common sector field equations (7.9), (7.10) and (7.11), together with (7.8), imply the identity

$$
\tilde{\nabla}^{i} \tilde{\nabla}_{i}\left(e^{-2 \phi}\left\|\hat{\eta}_{-}\right\|^{2}\right)=\frac{1}{6} e^{-2 \phi}\left\|\hat{\eta}_{-}\right\|^{2} \tilde{\mathcal{H}}_{i j k} \tilde{\mathcal{H}}^{i j k}
$$


Applying the maximum principle, one finds that $\mathcal{H}=0$, and

$$
\tilde{\nabla}_{i}\left(e^{-2 \phi}\left\|\hat{\eta}_{-}\right\|^{2}\right)=0
$$

which, together with (7.8) also implies

$$
h=-2 d \phi .
$$

On substituting this condition into (7.9), an additional application of the maximum principle implies that $\phi$ is constant, and $h=0$. So, if $\operatorname{Ker} \Theta_{-} \neq\{0\}$ then all the fluxes vanish.

Next, one can show that if $\hat{\eta}_{-}$solves the KSEs (7.8), then

$$
\hat{\eta}_{+}=\Gamma_{+} \Theta_{-} \hat{\eta}_{-},
$$

also solves (7.8), using the same type of reasoning as in the IIB analysis. The number of Killing spinors of common sector horizons is $N=\hat{N}+\check{N}$ with $\hat{N}=\hat{N}_{+}+\hat{N}_{-}$, where $\hat{N}_{ \pm}$ is the number of $\hat{\eta}_{ \pm}$Killing spinors, and similarly for $\tilde{N}$. We have therefore shown that, if $\hat{N} \neq 0$, then $\hat{N}_{+} \neq 0$.

To proceed further, the field equations (7.9), (7.10) and (7.11), together with (7.8) imply that

$$
\tilde{\nabla}^{i} \tilde{\nabla}_{i}\left(\left\|\hat{\eta}_{+}\right\|^{2}\right)-\left(2 \tilde{\nabla}^{i} \phi+h^{i}\right) \tilde{\nabla}_{i}\left(\left\|\hat{\eta}_{+}\right\|^{2}\right)=0,
$$

where we have also used the condition

$$
\left\|\hat{\eta}_{+}\right\|^{2}\left(\Delta-\frac{1}{4} S^{2}+\frac{1}{4} h^{2}\right)=0,
$$

which follows from the KSE. An application of the maximum principle gives that

$$
\tilde{\nabla}_{i}\left(\left\|\hat{\eta}_{+}\right\|^{2}\right)=0
$$

Then (7.8) and (7.19) imply that

$$
S=h, \quad \Delta=0 .
$$

The conditions $S=h$ and $\Delta=0$ are the same as those explored in [24] for heterotic horizons. So the common sector horizons can be investigated as special cases of the heterotic ones in [24]. In particular, one finds that

$$
\hat{\tilde{\nabla}} h=0,
$$

so $h$ generates an isometry on $\mathcal{S}$, and $h^{2}$ is constant. If $h=0$ then all the fluxes vanish.

We have proven that $\hat{N}_{+} \neq 0$ if $\hat{N} \neq 0$. It is straightforward to show that $\hat{N}_{-} \neq 0$, if $\hat{N} \neq 0$, as well. This is because, as was noted in [24], if $\hat{\eta}_{+}$solves the KSEs, then

$$
\hat{\eta}_{-}=\Gamma_{-} h_{i} \Gamma^{i} \hat{\eta}_{+},
$$


also solves the KSEs, so $\hat{N}_{-} \neq 0$ and the horizons preserve at least two supersymmetries.

In addition, a consequence of our analysis above is that the index of the Dirac operator on all common sector horizon sections vanishes. This is because $\Theta_{-}=\frac{1}{2} h_{i} \Gamma^{i}$ has an inverse $^{11}$ and pairs the zero modes of the $\Gamma^{i} \hat{\tilde{\nabla}}_{i}$ operator and its adjoint. On all common sector sections, the $\hat{A}$-genus vanishes. This can also be seen from the property of horizon sections to admit an isometry generated by $h$. An exhaustive analysis of the geometry of common sector horizons with extended supersymmetry can be done using the method of [24] applied for heterotic horizons. As a result all common sector horizons preserve an even number of supersymmetries, as $\hat{N}=\hat{N}_{+}+\hat{N}_{-}=2 \hat{N}_{-}$and similarly for $\tilde{N}$, and from the classification results of [29], if $\hat{N}_{-}>8$, then they are maximally supersymmetric with horizon sections isometric to $T^{8}$.

\section{Conclusions}

We have demonstrated that the number of supersymmetries $N$ preserved by IIB black hole horizons can be expressed in terms of the index of the Dirac operator on the horizon sections as in (1.1). As a consequence of this formula, IIB horizons preserve an even number of supersymmetries. Moreover if $N_{-} \neq 0$, the horizons with non-trivial fluxes exhibit an $\mathfrak{s l}(2, \mathbb{R})$ symmetry subalgebra. Furthermore, if the orbit of $\mathfrak{s l}(2, \mathbb{R})$ is 2-dimensional, then all such IIB horizons with non-trvial fluxes are warped products $A d S_{2} \times_{w} \mathcal{S}$. The proof of these results is based on the smoothness of the horizons, the compactness of the horizon sections, as well as the demonstration of Lichnerowicz type theorems which relate the Killing spinors to the zero modes of horizon Dirac operators. Instrumental in the proof are the field equations and Bianchi identities of the theory, and so the symmetry enhancement exhibited is dynamical. In addition, if $N_{-}=0$, the number of supersymmetries preserved by the horizons is given by the index of a Dirac operator, and so it is a topological invariant of the horizon sections. As a result, one can a priori test whether a manifold $\mathcal{S}$ with given Pontryagin numbers can admit a metric and fluxes such that it can identified as a IIB horizon section preserving a given number $N$ of supersymmetries.

The expression (1.1) for the number of supersymmetries $N$ of IIB black hole horizons also applies to M-horizons and horizons of the 5-dimensional minimal gauged supergravity as in the last two cases the index of the associated analogous horizon Dirac operator vanishes. The similarities in the proof of (1.1) for all horizons so far, including IIB and M-horizons, suggests that this formula is universal and applies to all supergravity theories. This is further supported by the observation that the IIB KSEs have a structure that encompasses that of the KSEs of all other supergravity theories, i.e. it has both a parallel transport equation associated with gravitino supersymmetry transformation and an algebraic KSE associated with the supersymmetry transformation of the dilatino. Therefore for $D \geq 4$ supergravity theories with standard matter couplings, we shall propose the following.

- The number of supersymmetries preserved by supergravity horizons is given by

$$
N=2 N_{-}+\operatorname{index}\left(D_{E}\right)
$$

\footnotetext{
${ }^{11}$ We have not been able to establish a similar property for all IIB horizons.
} 
where $N_{-}$is the dimension of the Kernel of a horizon Dirac operator which depends on the fluxes, and $D_{E}$ is the Dirac operator defined on an appropriate spinor bundle on the horizon sections $\mathcal{S}$ and twisted with $E$, and where $E$ is an appropriate vector bundle associated with the internal symmetries of the supergravity theory.

- All supergravity horizons with $N_{-} \neq 0$ and non-trivial fluxes admit an $\mathfrak{s l}(2, \mathbb{R})$ symmetry subalgebra. Furthermore, if the orbit of $\mathfrak{s l}(2, \mathbb{R})$ is 2-dimensional, then they are warped $A d S_{2}$ products.

It is not a priori apparent that index $\left(D_{E}\right)$ will be an even number but in all examples investigated so far the index is either an even number or it vanishes. Since the index vanishes on odd-dimensional manifolds, the proposed formula implies that all odd-dimensional supergravity horizons preserve an even number of supersymmetries, and if they have nontrivial fluxes it is likely that they will admit an $\mathfrak{s l}(2, \mathbb{R})$ symmetry subalgebra. It is also expected that the index vanishes for non-chiral even-dimensional supergravities and so again the associated horizons preserve even number of supersymmetries and admit an $\mathfrak{s l}(2, \mathbb{R})$ symmetry subalgebra.

Furthermore, the existence of an $\mathfrak{s l}(2, \mathbb{R})$ symmetry subalgebra of supergravity horizons is closely related to the presence of non-trivial fluxes. Therefore the existence of the $\mathfrak{s l}(2, \mathbb{R})$ symmetry is a property of the supergravity, and consequently a property of the low energy approximation of string theory and M-theory. It is also an indication that in the context of the AdS/CFT correspondence all such horizons have a conformal field theory dual.

\section{Acknowledgements}

UG is supported by the Knut and Alice Wallenberg Foundation. JG is supported by the STFC grant, ST/1004874/1. GP is partially supported by the STFC rolling grant ST/J002798/1.

\section{Appendix A Horizon Bianchi identities and field equations}

The Bianchi identities of IIB supergravity imply that

$$
X=d_{h} Y-\frac{i}{8}(\Phi \wedge \bar{H}-\bar{\Phi} \wedge H), \quad L=d_{h} \Phi-i \Lambda \wedge \Phi+\xi \wedge \bar{\Phi} .
$$

The self-duality of $F$ requires that

$$
X=-*_{8} X .
$$

The remaining Bianchi identities are

$$
\begin{aligned}
d \star_{8} Y & =\frac{i}{8} H \wedge \bar{H}, \quad d H=i \Lambda \wedge H-\xi \wedge \bar{H}, \\
d \xi & =2 i \Lambda \wedge \xi, \quad d \Lambda=-i \xi \wedge \bar{\xi}
\end{aligned}
$$


where $\Lambda$ is a $U(1)$ connection of $\lambda$, see [18] for more details.

The independent field equations of IIB horizons are

$$
\begin{gathered}
\tilde{\nabla}^{i} \Phi_{i}-i \Lambda^{i} \Phi_{i}-\xi^{i} \bar{\Phi}_{i}+\frac{2 i}{3} Y_{\ell_{1} \ell_{2} \ell_{3}} H^{\ell_{1} \ell_{2} \ell_{3}}=0 \\
\tilde{\nabla}^{\ell} H_{\ell i j}-i \Lambda^{\ell} H_{\ell i j}-h^{\ell} H_{\ell i j}+L_{i j}-\xi^{\ell} \bar{H}_{\ell i j}+\frac{2 i}{3}\left(\star_{8} Y_{i j \ell_{1} \ell_{2} \ell_{3}} H^{\ell_{1} \ell_{2} \ell_{3}}-6 Y_{i j \ell} \Phi^{\ell}\right)=0 \\
\tilde{\nabla}^{i} \xi_{i}-2 i \Lambda^{i} \xi_{i}-h^{i} \xi_{i}+\frac{1}{24}\left(-6 \Phi^{i} \Phi_{i}+H_{\ell_{1} \ell_{2} \ell_{3}} H^{\ell_{1} \ell_{2} \ell_{3}}\right)=0 \\
\frac{1}{2} \tilde{\nabla}^{i} h_{i}-\Delta-\frac{1}{2} h^{2}+\frac{2}{3} Y_{\ell_{1} \ell_{2} \ell_{3}} Y^{\ell_{1} \ell_{2} \ell_{3}}+\frac{3}{8} \Phi^{i} \bar{\Phi}_{i}+\frac{1}{48} H_{\ell_{1} \ell_{2} \ell_{3}} \bar{H}^{\ell_{1} \ell_{2} \ell_{3}}=0
\end{gathered}
$$

and

$$
\begin{aligned}
\tilde{R}_{i j}+\tilde{\nabla}_{(i} h_{j)}- & \frac{1}{2} h_{i} h_{j}+4 Y_{i \ell_{1} \ell_{2}} Y_{j}^{\ell_{1} \ell_{2}}+\frac{1}{2} \Phi_{(i} \bar{\Phi}_{j)}-2 \xi_{(i} \bar{\xi}_{j)}-\frac{1}{4} H_{\ell_{1} \ell_{2}(i} \bar{H}_{j)} \ell_{1} \ell_{2} \\
& +\delta_{i j}\left(-\frac{1}{8} \Phi_{\ell} \bar{\Phi}^{\ell}-\frac{2}{3} Y_{\ell_{1} \ell_{2} \ell_{3}} Y^{\ell_{1} \ell_{2} \ell_{3}}+\frac{1}{48} H_{\ell_{1} \ell_{2} \ell_{3}} \bar{H}^{\ell_{1} \ell_{2} \ell_{3}}\right)=0
\end{aligned}
$$

where $\tilde{R}$ is the Ricci tensor of $\mathcal{S}$. There are three additional field equations which are not independent because they follow from those above. These are

$$
\begin{gathered}
-\tilde{\nabla}^{i} L_{i m}+i \Lambda^{i} L_{i m}+h^{i} L_{i m}-\frac{1}{2} d h^{i j} H_{i j m}+\xi^{i} \bar{L}_{i m}+\frac{2 i}{3}\left(X_{m \ell_{1} \ell_{2} \ell_{3}} H^{\ell_{1} \ell_{3} \ell_{3}}-3 Y_{m \ell_{1} \ell_{2}} L^{\ell_{1} \ell_{2}}\right)=0 \\
-\frac{1}{2} \tilde{\nabla}^{j} d h_{j i}-d h_{i j} h^{j}-\tilde{\nabla}_{i} \Delta+\Delta h_{i}+\frac{4}{3} X_{i \ell_{1} \ell_{2} \ell_{3}} Y^{\ell_{1} \ell_{2} \ell_{3}} \\
-\frac{1}{8}\left(L_{\ell_{1} \ell_{2}} \bar{H}_{i}^{\ell_{1} \ell_{2}}-2 \Phi^{\ell} \bar{L}_{i \ell}+\bar{L}_{\ell_{1} \ell_{2}} H_{i}^{\ell_{1} \ell_{2}}-2 \bar{\Phi}^{\ell} L_{i \ell}\right)=0
\end{gathered}
$$

and

$\frac{1}{2} \tilde{\nabla}^{2} \Delta-\frac{3}{2} h^{i} \tilde{\nabla}_{i} \Delta-\frac{1}{2} \Delta \hat{\nabla}^{i} h_{i}+\Delta h^{2}+\frac{1}{4} d h_{i j} d h^{i j}-\frac{1}{6} X_{\ell_{1} \ell_{2} \ell_{3} \ell_{4}} X^{\ell_{1} \ell_{2} \ell_{3} \ell_{4}}-\frac{1}{4} L_{i j} \bar{L}^{i j}=0$,

which we state because they are useful in the investigation of the KSEs. 


\section{Appendix B Independent KSEs}

It is well known that the KSEs imply some of the Bianchi identities and field equations of a theory. Because of this, to find solutions it is customary to solve the KSEs and then impose the remaining field equations and Bianchi identities. However, we shall not do this here because of the complexity of solving the KSEs (2.8), (2.9), (2.13), and (2.16) which contain the $\tau$ spinor as expressed in (2.11). Instead, we shall first show that all the KSEs which contain $\tau_{+}$are actually implied from those containing $\phi_{+}$, i.e. $(2.12)$ and (2.15), and some of the field equations and Bianchi identities.

Then we also show that (2.10) and the terms linear in $u$ in (2.12) and (2.15) are implied by the field equations, Bianchi identities and (2.14) and (2.17).

\section{B.1 The (2.13) condition}

The (2.13) component of KSEs is implied by (2.12) and (2.11) together with a number of bosonic field equations and Bianchi identities. To see this, first evaluate the LHS of (2.13) by substituting in (2.11) to eliminate $\tau_{+}$, and use (2.12) to evaluate the supercovariant derivatives of $\eta_{+}$and $C * \eta_{+}$. Also evaluate

$$
\begin{array}{r}
\left(\frac{1}{4} \tilde{R}_{i j} \Gamma^{j}-\frac{1}{2} \Gamma^{j}\left(\tilde{\nabla}_{j} \tilde{\nabla}_{i}-\tilde{\nabla}_{i} \tilde{\nabla}_{j}\right)\right) \phi_{+}-\frac{1}{2} \xi_{i} C * \mathcal{A}_{1} \\
-\left(\frac{1}{192} \Gamma_{i}^{\ell_{1} \ell_{2} \ell_{3}} \bar{H}_{\ell_{1} \ell_{2} \ell_{3}}-\frac{3}{64} \bar{H}_{i \ell_{1} \ell_{2}} \Gamma^{\ell_{1} \ell_{2}}-\frac{1}{32} \Gamma_{i}^{\ell} \bar{\Phi}_{\ell}+\frac{3}{32} \bar{\Phi}_{i}\right) \mathcal{A}_{1}=0
\end{array}
$$

where

$$
\mathcal{A}_{1}=\left(-\frac{1}{4} \Phi_{i} \Gamma^{i}+\frac{1}{24} H_{\ell_{1} \ell_{2} \ell_{3}} \Gamma^{\ell_{1} \ell_{2} \ell_{3}}\right) \phi_{+}+\xi_{i} \Gamma^{i} C * \phi_{+} .
$$

The expression in (B.1) vanishes on making use of (2.15), as $\mathcal{A}_{1}=0$ is equivalent to (2.15). However a non-trivial identity is obtained by expanding out the supercovariant derivative terms again using (2.12), and expanding out the $\mathcal{A}_{1}$ terms using (B.2). Then,

on adding (B.1) to the LHS of (2.13), with $\tau_{+}$eliminated in favour of $\eta_{+}$using (2.11) and (2.12) as mentioned above, one obtains, after some calculation, a term proportional to (A.8).

Therefore, it follows that (2.13) is implied by (2.12) and (2.15) and (2.11), and the bosonic field equations and Bianchi identities. We remark that in addition to using (A.8) in establishing this identity, we also make use of (A.3), (A.2), (A.4) and (A.5).

\section{B.2 The (2.16) condition}

Next consider (2.15) and (2.16). On defining

$$
\mathcal{A}_{2}=\left(-\frac{1}{4} \Phi_{i} \Gamma^{i}-\frac{1}{24} H_{\ell_{1} \ell_{2} \ell_{3}} \Gamma^{\ell_{1} \ell_{2} \ell_{3}}\right) \tau_{+}-\xi_{i} \Gamma^{i} C * \tau_{+}+\frac{1}{8} L_{i j} \Gamma^{i j} \phi_{+},
$$


one obtains the following identity

$$
\mathcal{A}_{2}=-\frac{1}{2} \Gamma^{i} \tilde{\nabla}_{i} \mathcal{A}_{1}+\left(\frac{3 i}{4} \Lambda_{i} \Gamma^{i}+\frac{3}{8} h_{i} \Gamma^{i}-\frac{i}{12} Y_{\ell_{1} \ell_{2} \ell_{3}} \Gamma^{\ell_{1} \ell_{2} \ell_{3}}\right) \mathcal{A}_{1},
$$

where we have made use of (2.12) in order to evaluate the covariant derivative in the above expression. In addition, we also have made use of the following field equations and Bianchi identities: (A.3), (A.4), (A.5) and (A.6). It follows that these conditions, together with (2.15) imply (2.16).

\section{B.3 The (2.8) condition}

To show that (2.8) is also implied for the KSEs involving only $\eta$ and the field equations and Bianchi identities, contract (2.13) with $\Gamma^{i}$ and use (2.11) to rewrite the $\tau_{+}$terms in terms of $\phi_{+}$. Then subtract $\left(\frac{3}{16} \bar{\Phi}_{i} \Gamma^{i}+\frac{1}{96} \bar{H}_{\ell_{1} \ell_{2} \ell_{3}} \Gamma^{\ell_{1} \ell_{2} \ell_{3}}\right) \mathcal{A}_{1}$ from the resulting expression to obtain (2.8). In order to obtain (2.8) from these expressions, we also make use of (A.3), (A.2), (A.5), (A.4), and (A.7). It follows, from section B.1 above, that (2.8) follows from the above mentioned Bianchi identities and field equations, together with (2.12) and (2.15).

\section{B.4 The (2.9) condition}

The (2.9) condition is obtained from (2.8) as follows. First act on (2.8) with the Dirac operator $\Gamma^{i} \tilde{\nabla}_{i}$, and use the bosonic field equations and Bianchi identities to eliminate the $d \star_{8} d h, d L, d \star_{8} L, d \star_{8} h, d Y, d \star_{8} Y, d H$ and $d \star_{8} H$ terms, and rewrite $d \Phi$ in terms of $L$. Then use the algebraic conditions (2.15) and (2.16) to eliminate the $\xi$-terms from the resulting expression. The terms involving $\Lambda$ then vanish as a consequence of (2.8).

Next consider the $d h$-terms; after some calculation, these can be rewritten as

$$
\frac{1}{2} d h_{i j} \Gamma^{i j} \tau_{+}-\frac{7}{32} h_{\ell} \Gamma^{\ell} d h_{i j} \Gamma^{i j} \phi_{+}+\left(-\frac{1}{64} \Phi_{\ell} \Gamma^{\ell}+\frac{1}{384} H_{\ell_{1} \ell_{2} \ell_{3}} \Gamma^{\ell_{1} \ell_{2} \ell_{3}}\right) d h_{i j} \Gamma^{i j} C * \phi_{+} .
$$

The $d h$ terms involving $\phi_{+}$and $C * \phi_{+}$in the above expression are then eliminated, using (2.8). On collating the remaining terms, one finds that those involving $\Delta$ (but not $d \Delta$ ) are

$$
-\Delta h_{j} \Gamma^{j} \phi_{+}
$$

It is also straightforward to note that the terms involving $\bar{L}$ vanish, whereas the terms involving $X$ and $L$ can be rewritten as

$$
-\frac{1}{2} L_{i j} \Gamma^{i j} C * \tau_{+}-\frac{i}{12} X_{\ell_{1} \ell_{2} \ell_{3} \ell_{4}} \Gamma^{\ell_{1} \ell_{2} \ell_{3} \ell_{4}} \tau_{+}
$$

where the anti-self-duality of $X$ has been used to simplify the expression. The remaining terms which are linear in $\tau_{+}, C * \tau_{+}$and quadratic in $h, Y, \Phi, \bar{\Phi}, H, \bar{H}$ can be shown to vanish after some computation. After performing these calculations, the condition which is obtained is (2.9). 


\section{B.5 The (2.15) condition}

Next consider the part of (2.15) which is linear in $u$. On defining

$$
\mathcal{B}_{1}=\left(\frac{1}{4} \Phi_{i} \Gamma^{i}+\frac{1}{24} H_{\ell_{1} \ell_{2} \ell_{3}} \Gamma^{\ell_{1} \ell_{2} \ell_{3}}\right) \eta_{-}+\xi_{i} \Gamma^{i} C * \eta_{-},
$$

one finds that the $u$-dependent part of (2.15) is proportional to

$$
-\frac{1}{2} \Gamma^{i} \tilde{\nabla}_{i} \mathcal{B}_{1}+\left(\frac{3 i}{4} \Lambda_{i} \Gamma^{i}+\frac{1}{8} h_{i} \Gamma^{i}+\frac{i}{12} Y_{\ell_{1} \ell_{2} \ell_{3}} \Gamma^{\ell_{1} \ell_{2} \ell_{3}}\right) \mathcal{B}_{1}
$$

where we have made use of (2.14) in order to evaluate the covariant derivative in the above expression. In addition, we also have made use of the following field equations and Bianchi identities: (A.3), (A.4), (A.5) and (A.6). It follows that these conditions, together with (2.17) imply the $u$-dependent part of (2.15).

\section{B.6 The (2.10) condition}

Next, consider (2.10). To show that this condition is implied by $(2.14),(2.17)$ and the bosonic conditions, compute

$$
\left(\Gamma^{i} \Gamma^{j}\left(\tilde{\nabla}_{j} \tilde{\nabla}_{i}-\tilde{\nabla}_{i} \tilde{\nabla}_{j}\right)-\frac{1}{2} \tilde{R}\right) \eta_{-}+\xi_{i} \Gamma^{i} C * \mathcal{B}_{1}+\left(\frac{5}{8} \bar{\Phi}_{\ell} \Gamma^{\ell}-\frac{1}{16} \bar{H}_{\ell_{1} \ell_{2} \ell_{3}} \Gamma^{\ell_{1} \ell_{2} \ell_{3}}\right) \mathcal{B}_{1}
$$

where we use (2.14) to evaluate the covariant derivative terms, and (2.17) implies that the terms involving $\mathcal{B}_{1}$ vanish. After making use of the field equations and Bianchi identities (specifically, (A.3), (A.4), (A.5), (A.7) and (A.8)), one obtains after some calculation, a term proportional to $(2.10)$.

\section{B.7 The (2.12) condition}

We next consider the part of (2.12) which is linear in $u$. First compute

$$
\begin{array}{r}
\left(\Gamma^{j}\left(\tilde{\nabla}_{j} \tilde{\nabla}_{i}-\tilde{\nabla}_{i} \tilde{\nabla}_{j}\right) \eta_{-}-\frac{1}{2} \Gamma^{j} \tilde{R}_{i j} \eta_{-}\right)+\xi_{i} C * \mathcal{B}_{1} \\
-\left(-\frac{1}{16} \Gamma_{i}{ }^{q} \bar{\Phi}_{q}+\frac{3}{16} \bar{\Phi}_{i}-\frac{1}{96} \Gamma_{i}^{\ell_{1} \ell_{2} \ell_{3}} \bar{H}_{\ell_{1} \ell_{2} \ell_{3}}+\frac{3}{32} \bar{H}_{i \ell_{1} \ell_{2}} \Gamma^{\ell_{1} \ell_{2}}\right) \mathcal{B}_{1}
\end{array}
$$

which vanishes identically as a consequence of (2.10). In particular, use (2.14) to evaluate the covariant derivative terms, also using the field equations and Bianchi identities (specifically, (A.3), (A.2), (A.4), (A.5), (A.7) and (A.8)).

The resulting identity obtained from (B.10) corresponds to the expression obtained by expanding out the $u$-dependent part of (2.12), again using (2.14) to evaluate the covariant derivative terms. Hence the $u$-dependent part of (2.12) is implied by (2.14) and (2.10) together with the field equations and Bianchi identities. 


\section{Appendix C Lichnerowicz Theorems}

\section{C.1 Proof of the maximum principle on $\left\|\eta_{+}\right\|^{2}$}

In this appendix we shall give the proof of the Lichnerowicz type theorem described in section 3.1. Throughout the following analysis, we assume all of the field equations and Bianchi identities of IIB supergravity listed in appendix A.

To proceed, we rewrite the KSE (2.18) as

$$
\nabla_{i}^{(+)} \eta_{+} \equiv \tilde{\nabla}_{i} \eta_{+}+\psi_{i}^{(+)} \eta_{+}+\theta_{i}^{(+)} C * \eta_{+}=0
$$

where

$$
\psi_{i}^{(+)}=-\frac{i}{2} \Lambda_{i}-\frac{1}{4} h_{i}-\frac{i}{4} Y_{i \ell_{1} \ell_{2}} \Gamma^{\ell_{1} \ell_{2}}+\frac{i}{12} \Gamma_{i}^{\ell_{1} \ell_{2} \ell_{3}} Y_{\ell_{1} \ell_{2} \ell_{3}}
$$

and

$$
\theta_{i}^{(+)}=\frac{1}{16} \Gamma_{i}^{j} \Phi_{j}-\frac{3}{16} \Phi_{i}-\frac{1}{96} \Gamma_{i}^{\ell_{1} \ell_{2} \ell_{3}} H_{\ell_{1} \ell_{2} \ell_{3}}+\frac{3}{32} H_{i \ell_{1} \ell_{2}} \Gamma^{\ell_{1} \ell_{2}}
$$

We also rewrite the associated horizon Dirac equation (3.1) as

$$
\mathcal{D}^{(+)} \eta_{+} \equiv \Gamma^{i} \tilde{\nabla}_{i} \eta_{+}+\psi^{(+)} \eta_{+}+\theta^{(+)} C * \eta_{+}=0
$$

with

$$
\psi^{(+)}=\Gamma^{i} \psi_{i}^{(+)}=-\frac{i}{2} \Lambda_{i} \Gamma^{i}-\frac{1}{4} h_{i} \Gamma^{i}+\frac{i}{6} Y_{\ell_{1} \ell_{2} \ell_{3}} \Gamma^{\ell_{1} \ell_{2} \ell_{3}}
$$

and

$$
\theta^{(+)}=\Gamma^{i} \theta_{i}^{(+)}=\frac{1}{4} \Phi_{i} \Gamma^{i}+\frac{1}{24} H_{\ell_{1} \ell_{2} \ell_{3}} \Gamma^{\ell_{1} \ell_{2} \ell_{3}}
$$

Assume that the horizon Dirac equation (C.4) holds, we compute the Laplacian

$$
\left.\tilde{\nabla}^{i} \tilde{\nabla}_{i}\left\langle\eta_{+}, \eta_{+}\right\rangle=2 \operatorname{Re}\left(\left\langle\eta_{+}, \tilde{\nabla}^{i} \tilde{\nabla}_{i} \eta_{+}\right\rangle\right)+2\left\langle\tilde{\nabla}_{i} \eta_{+}, \tilde{\nabla}^{i} \eta_{+}\right\rangle\right)
$$

To evaluate this expression note that

$$
\begin{aligned}
\tilde{\nabla}^{i} \tilde{\nabla}_{i} \eta_{+} & =\Gamma^{i} \tilde{\nabla}_{i}\left(\Gamma^{j} \tilde{\nabla}_{j} \eta_{+}\right)+\frac{1}{4} \tilde{R} \eta_{+} \\
& =\Gamma^{i} \tilde{\nabla}_{i}\left(-\psi^{(+)} \eta_{+}-\theta^{(+)} C * \eta_{+}\right) \\
& +\frac{1}{4}\left(-\tilde{\nabla}^{i} h_{i}+\frac{1}{2} h^{2}+\frac{4}{3} Y_{\ell_{1} \ell_{2} \ell_{3}} Y^{\ell_{1} \ell_{2} \ell_{3}}+\frac{1}{2} \Phi_{i} \bar{\Phi}^{i}+2 \xi_{i} \bar{\xi}^{i}+\frac{1}{12} H_{\ell_{1} \ell_{2} \ell_{3}} \bar{H}^{\ell_{1} \ell_{2} \ell_{3}}\right)\left\langle\eta_{+}, \eta_{+}\right\rangle
\end{aligned}
$$


It follows that

$$
\begin{aligned}
\operatorname{Re}\left(\left\langle\eta_{+}, \tilde{\nabla}^{i} \tilde{\nabla}_{i} \eta_{+}\right\rangle\right) & =\frac{1}{4}\left(-\tilde{\nabla}^{i} h_{i}+\frac{1}{2} h^{2}+\frac{4}{3} Y_{\ell_{1} \ell_{2} \ell_{3}} Y^{\ell_{1} \ell_{2} \ell_{3}}\right. \\
& \left.+\frac{1}{2} \Phi_{i} \bar{\Phi}^{i}+2 \xi_{i} \bar{\xi}^{i}+\frac{1}{12} H_{\ell_{1} \ell_{2} \ell_{3}} \bar{H}^{\ell_{1} \ell_{2} \ell_{3}}\right)\left\langle\eta_{+}, \eta_{+}\right\rangle \\
& +\left\langle\eta_{+},\left(\frac{1}{4} \tilde{\nabla}^{i} h_{i}-\frac{i}{2} \tilde{\nabla}^{i} Y_{i \ell_{1} \ell_{2}} \Gamma^{\ell_{1} \ell_{2}}+\frac{i}{4}(d \Lambda)_{i j} \Gamma^{i j}\right) \eta_{+}\right\rangle \\
& +\operatorname{Re}\left\langle\eta_{+},\left(-\frac{1}{4} \tilde{\nabla}^{i} \Phi_{i}-\frac{1}{96}(d H)_{\ell_{1} \ell_{2} \ell_{3} \ell_{4}} \Gamma^{\ell_{1} \ell_{2} \ell_{3} \ell_{4}}\right) C * \eta_{+}\right\rangle \\
& +\operatorname{Re}\left\langle\eta_{+}, \Gamma^{i}\left(\frac{i}{2} \Lambda_{j} \Gamma^{j}+\frac{1}{4} h_{j} \Gamma^{j}-\frac{i}{6} Y_{\ell_{1} \ell_{2} \ell_{3}} \Gamma^{\ell_{1} \ell_{2} \ell_{3}}\right) \tilde{\nabla}_{i} \eta_{+}\right\rangle \\
& +\operatorname{Re}\left\langle\eta_{+}, \Gamma^{i}\left(-\frac{1}{4} \Phi_{j} \Gamma^{j}-\frac{1}{24} H_{\ell_{1} \ell_{2} \ell_{3}} \Gamma^{\ell_{1} \ell_{2} \ell_{3}}\right) C * \tilde{\nabla}_{i} \eta_{+}\right\rangle .
\end{aligned}
$$

Using the field equations and Bianchi identities, the terms involving $\tilde{\nabla}^{i} Y_{i \ell_{1} \ell_{2}}, d \Lambda, \tilde{\nabla}^{i} \Phi_{i}, d H$ can be rewritten as terms quadratic in fluxes to give

$$
\begin{aligned}
\operatorname{Re}\left(\left\langle\eta_{+}, \tilde{\nabla}^{i} \tilde{\nabla}_{i} \eta_{+}\right\rangle\right) & =\left(\frac{1}{8} h^{2}+\frac{1}{3} Y_{\ell_{1} \ell_{2} \ell_{3}} Y^{\ell_{1} \ell_{2} \ell_{3}}+\frac{1}{8} \Phi_{i} \bar{\Phi}^{i}+\frac{1}{2} \xi_{i} \bar{\xi}^{i}+\frac{1}{48} H_{\ell_{1} \ell_{2} \ell_{3}} \bar{H}^{\ell_{1} \ell_{2} \ell_{3}}\right)\left\langle\eta_{+}, \eta_{+}\right\rangle \\
& +\left\langle\eta_{+},\left(\frac{1}{288} H_{\ell_{1} \ell_{2} \ell_{3}} \bar{H}_{\ell_{4} \ell_{5} \ell_{6}} \Gamma^{\ell_{1} \ell_{2} \ell_{3} \ell_{4} \ell_{5} \ell_{6}}+\frac{1}{2} \xi_{\ell_{1}} \bar{\xi}_{\ell_{2}} \Gamma^{\ell_{1} \ell_{2}}\right) \eta_{+}\right\rangle \\
& +\operatorname{Re}\left\langle\eta_{+},\left(-\frac{i}{4} \Lambda^{i} \Phi_{i}-\frac{1}{4} \xi^{i} \bar{\Phi}_{i}+\frac{i}{6} Y_{\ell_{1} \ell_{2} \ell_{3}} H^{\ell_{1} \ell_{2} \ell_{3}}\right.\right. \\
& \left.\left.-\frac{i}{24} \Lambda_{\ell_{1}} H_{\ell_{1} \ell_{3} \ell_{4}} \Gamma^{\ell_{1} \ell_{2} \ell_{3} \ell_{4}}+\frac{1}{24} \xi_{\ell_{1}} \bar{H}_{\ell_{2} \ell_{3} \ell_{4}} \Gamma^{\ell_{1} \ell_{2} \ell_{3} \ell_{4}}\right) C * \eta_{+}\right\rangle \\
& +\operatorname{Re}\left\langle\eta_{+}, \Gamma^{i}\left(\frac{i}{2} \Lambda_{j} \Gamma^{j}+\frac{1}{4} h_{j} \Gamma^{j}-\frac{i}{6} Y_{\ell_{1} \ell_{2} \ell_{3}} \Gamma^{\ell_{1} \ell_{2} \ell_{3}}\right) \tilde{\nabla}_{i} \eta_{+}\right\rangle \\
& +\operatorname{Re}\left\langle\eta_{+}, \Gamma^{i}\left(-\frac{1}{4} \Phi_{j} \Gamma^{j}-\frac{1}{24} H_{\ell_{1} \ell_{2} \ell_{3}} \Gamma^{\ell_{1} \ell_{2} \ell_{3}}\right) C * \tilde{\nabla}_{i} \eta_{+}\right\rangle, \quad \text { (C.10) }
\end{aligned}
$$

where we have made use of the identities

$$
\left\langle\eta_{+}, \Gamma_{i j} C * \eta_{+}\right\rangle=0 .
$$

Having evaluated the first term in (C.7), we compute the second term, writing

$$
\begin{aligned}
\left\langle\tilde{\nabla}^{i} \eta_{+}, \tilde{\nabla}_{i} \eta_{+}\right\rangle & =\left\langle\tilde{\nabla}^{i} \eta_{+}+\psi^{(+) i} \eta_{+}+\theta^{(+) i} C * \eta_{+}, \tilde{\nabla}_{i} \eta_{+}+\psi_{i}^{(+)} \eta_{+}+\theta_{i}^{(+)} C * \eta_{+}\right\rangle \\
& -\left\langle\psi^{(+) i} \eta_{+}+\theta^{(+) i} C * \eta_{+}, \psi_{i}^{(+)} \eta_{+}+\theta_{i}^{(+)} C * \eta_{+}\right\rangle \\
& +\operatorname{Re}\left\langle\eta_{+},-2\left(\psi^{(+) i}\right)^{\dagger} \tilde{\nabla}_{i} \eta_{+}-2\left(C *\left(\theta^{(+) i}\right)^{\dagger}\right) \tilde{\nabla}_{i} C * \eta_{+}\right\rangle
\end{aligned}
$$

The first term on the RHS is the norm squared of the horizon KSE (2.12). Next, combine the terms which involve $\tilde{\nabla}_{i} \eta_{+}$and $\tilde{\nabla}_{i} C * \eta_{+}$in the last two lines of (C.10) and the last line of (C.12). These can be rewritten using the identities

$$
\Gamma^{i}\left(\frac{i}{2} \Lambda_{j} \Gamma^{j}+\frac{1}{4} h^{j} \Gamma^{j}-\frac{i}{6} Y_{\ell_{1} \ell_{2} \ell_{3}} \Gamma^{\ell_{1} \ell_{2} \ell_{3}}\right)-2\left(\psi^{(+) i}\right)^{\dagger}=h^{i}+\left(-\frac{i}{2} \Lambda^{j} \Gamma^{j}-\frac{1}{4} h_{j} \Gamma^{j}\right) \Gamma^{i},
$$


and

$$
\Gamma^{i}\left(-\frac{1}{4} \Phi_{j} \Gamma^{j}-\frac{1}{24} H_{\ell_{1} \ell_{2} \ell_{3}} \Gamma^{\ell_{1} \ell_{2} \ell_{3}}\right)-2\left(C *\left(\theta^{(+) i}\right)^{\dagger}\right)=\left(\frac{1}{8} \Phi_{j} \Gamma^{j}+\frac{1}{48} H_{\ell_{1} \ell_{2} \ell_{3}} \Gamma^{\ell_{1} \ell_{2} \ell_{3}}\right) \Gamma^{i}
$$

It follows that the sum of the last two lines in (C.10) and the last line in (C.12) can be rewritten, using the horizon Dirac equation, to give

$$
\begin{aligned}
& \operatorname{Re}\left\langle\eta_{+}, \Gamma^{i}\left(\frac{i}{2} \Lambda_{j} \Gamma^{j}+\frac{1}{4} h_{j} \Gamma^{j}-\frac{i}{6} Y_{\ell_{1} \ell_{2} \ell_{3}} \Gamma^{\ell_{1} \ell_{2} \ell_{3}}\right) \tilde{\nabla}_{i} \eta_{+}\right\rangle \\
+ & \operatorname{Re}\left\langle\eta_{+}, \Gamma^{i}\left(-\frac{1}{4} \Phi_{j} \Gamma^{j}-\frac{1}{24} H_{\ell_{1} \ell_{2} \ell_{3}} \Gamma^{\ell_{1} \ell_{2} \ell_{3}}\right) C * \tilde{\nabla}_{i} \eta_{+}\right\rangle \\
+ & \operatorname{Re}\left\langle\eta_{+},-2\left(\psi^{(+) i}\right)^{\dagger} \tilde{\nabla}_{i} \eta_{+}-2\left(C *\left(\theta^{(+) i}\right)^{\dagger}\right) \tilde{\nabla}_{i} C * \eta_{+}\right\rangle \\
= & \frac{1}{2} h^{i} \tilde{\nabla}_{i}\left\langle\eta_{+}, \eta_{+}\right\rangle \\
+ & \operatorname{Re}\left\langle\eta_{+},\left(-\frac{i}{2} \Lambda^{j} \Gamma^{j}-\frac{1}{4} h_{j} \Gamma^{j}\right)\left(-\psi^{(+)} \eta_{+}-\theta^{(+)} C * \eta_{+}\right)\right\rangle \\
+ & \operatorname{Re}\left\langle\eta_{+},\left(\frac{1}{8} \Phi_{j} \Gamma^{j}+\frac{1}{48} H_{\ell_{1} \ell_{2} \ell_{3}} \Gamma^{\ell_{1} \ell_{2} \ell_{3}}\right)\left(-\left(C * \psi^{(+)}\right) C * \eta_{+}-\left(C * \theta^{(+)}\right) \eta_{+}\right)\right\rangle .
\end{aligned}
$$

So, to evaluate (C.7) one takes the sum of (C.10) and (C.12), using (C.15) to rewrite the last two lines in (C.10) and the last line in (C.12) as given above. On expanding out all of the terms quadratic in the fluxes, one obtains (3.5).

\section{C.2 Proof of a Lichnerowicz Theorem for $\mathcal{D}^{(-)}$}

In this appendix, we shall prove a Lichnerowicz identity for the $\mathcal{D}^{(-)}$operator in (3.1). We remark that throughout the following analysis, we will again assume all of the Bianchi identities and field equations which are listed in appendix A.

To proceed, we rewrite the KSE (2.18) as

$$
\nabla^{(-)} \eta_{-} \equiv \tilde{\nabla}_{i} \eta_{-}+\psi_{i}^{(-)} \eta_{-}+\theta_{i}^{(-)} C * \eta_{-}
$$

where

$$
\psi_{i}^{(-)}=-\frac{i}{2} \Lambda_{i}+\frac{1}{4} h_{i}+\frac{i}{4} Y_{i \ell_{1} \ell_{2}} \Gamma^{\ell_{1} \ell_{2}}-\frac{i}{12} \Gamma_{i}^{\ell_{1} \ell_{2} \ell_{3}} Y_{\ell_{1} \ell_{2} \ell_{3}}
$$

and

$$
\theta_{i}^{(-)}=-\frac{1}{16} \Gamma_{i}^{j} \Phi_{j}+\frac{3}{16} \Phi_{i}-\frac{1}{96} \Gamma_{i}^{\ell_{1} \ell_{2} \ell_{3}} H_{\ell_{1} \ell_{2} \ell_{3}}+\frac{3}{32} H_{i \ell_{1} \ell_{2}} \Gamma^{\ell_{1} \ell_{2}}
$$

We also rewrite the associated horizon Dirac equation (3.1) as

$$
\mathcal{D}^{(-)} \eta_{-} \equiv \Gamma^{i} \tilde{\nabla}_{i} \eta_{-}+\psi^{(-)} \eta_{-}+\theta^{(-)} C * \eta_{-},
$$


with

$$
\psi^{(-)}=\Gamma^{i} \psi_{i}^{(-)}=-\frac{i}{2} \Lambda_{i} \Gamma^{i}+\frac{1}{4} h_{i} \Gamma^{i}-\frac{i}{6} Y_{\ell_{1} \ell_{2} \ell_{3}} \Gamma^{\ell_{1} \ell_{2} \ell_{3}},
$$

and

$$
\theta^{(-)}=\Gamma^{i} \theta_{i}^{(-)}=-\frac{1}{4} \Phi_{i} \Gamma^{i}+\frac{1}{24} H_{\ell_{1} \ell_{2} \ell_{3}} \Gamma^{\ell_{1} \ell_{2} \ell_{3}}
$$

We define

$$
\mathcal{I}=\int_{\mathcal{S}}\left(\left\|\nabla^{(-)} \eta_{-}\right\|^{2}-\left\|\mathcal{D}^{(-)} \eta_{-}\right\|^{2}\right)
$$

and decompose

$$
\mathcal{I}=\mathcal{I}_{1}+\mathcal{I}_{2}+\mathcal{I}_{3}
$$

where

$$
\mathcal{I}_{1}=\int_{\mathcal{S}}\left\langle\tilde{\nabla}_{i} \eta_{-}, \tilde{\nabla}^{i} \eta_{-}\right\rangle-\left\langle\Gamma^{i} \tilde{\nabla}_{i} \eta_{-}, \Gamma^{j} \tilde{\nabla}_{j} \eta_{-}\right\rangle
$$

and

$$
\mathcal{I}_{2}=2 \operatorname{Re}\left(\int_{\mathcal{S}}\left\langle\tilde{\nabla}_{i} \eta_{-}, \Psi^{(-) i} \eta_{-}\right\rangle-\left\langle\Gamma^{i} \tilde{\nabla}_{i} \eta_{-}, \Psi^{(-)} \eta_{-}\right\rangle\right)
$$

and

$$
\mathcal{I}_{3}=\int_{\mathcal{S}}\left\langle\Psi_{i}^{(-)} \eta_{-}, \Psi^{(-) i} \eta_{-}\right\rangle-\left\langle\Psi^{(-)} \eta_{-}, \Psi^{(-)} \eta_{-}\right\rangle
$$

On using the identity

$$
\langle C * \eta, \tau\rangle=\overline{\langle\eta, C * \tau\rangle},
$$

for any $\eta, \tau$, it is straightforward to rewrite (C.26) as

$$
\begin{aligned}
\mathcal{I}_{3} & =\int_{\mathcal{S}}\left\langle\eta_{-},\left(\left(\psi_{i}^{(-)}\right)^{\dagger} \psi^{(-) i}-\psi^{(-) \dagger} \psi^{(-)}+C *\left(\left(\theta^{(-) i}\right)^{\dagger} \theta_{i}^{(-)}-\theta^{(-) \dagger} \theta^{(-)}\right)\right) \eta_{-}\right\rangle \\
& +2 \operatorname{Re} \int_{\mathcal{S}}\left\langle\eta_{-},\left(\left(\psi_{i}^{(-)}\right)^{\dagger} \theta^{(-) i}-\psi^{(-) \dagger} \theta^{(-)}\right) C * \eta_{-}\right\rangle
\end{aligned}
$$

where in the first line of the above expression, the charge conjugation acts solely on $\left(\theta^{(-) i}\right)^{\dagger} \theta_{i}^{(-)}-\theta^{(-) \dagger} \theta^{(-)}$. 
To proceed we expand out (C.28) to obtain

$$
\begin{aligned}
\mathcal{I}_{3} & =\int_{\mathcal{S}}\left\langle\eta_{-},\left(\frac{1}{6} Y_{\ell_{1} \ell_{2} \ell_{3}} Y^{\ell_{1} \ell_{2} \ell_{3}}+\frac{1}{96} H_{\ell_{1} \ell_{2} \ell_{3}} \bar{H}^{\ell_{1} \ell_{2} \ell_{3}}+\left(-\frac{i}{4} \Lambda_{\ell_{1}} h_{\ell_{2}}+\frac{3 i}{8} h^{i} Y_{i \ell_{1} \ell_{2}}\right.\right.\right. \\
& -\frac{1}{16} \Phi_{\ell_{1}} \bar{\Phi}_{\ell_{2}}+\frac{3}{64} \Phi^{i} \bar{H}_{i \ell_{1} \ell_{2}}-\frac{3}{64} \bar{\Phi}^{i} H_{i \ell_{1} \ell_{2}} \\
& \left.+\frac{1}{576} \epsilon_{\ell_{1} \ell_{2}}{ }^{q_{q_{2}} q_{3} q_{4} q_{5} q_{6}} H_{q_{1} q_{2} q_{3}} \bar{H}_{q_{4} q_{5} q_{6}}\right) \Gamma^{\ell_{1} \ell_{2}} \\
& +\left(-\frac{1}{12} \Lambda_{\ell_{1}} Y_{\ell_{2} \ell_{3} \ell_{4}}+\frac{1}{4} Y^{i}{ }_{\ell_{1} \ell_{2}} Y_{i \ell_{3} \ell_{4}}+\frac{1}{192} \Phi_{\ell_{1}} \bar{H}_{\ell_{2} \ell_{3} \ell_{4}}\right. \\
& \left.\left.\left.+\frac{1}{192} \bar{\varphi}_{\ell_{1}} H_{\ell_{2} \ell_{3} \ell_{4}}+\frac{1}{64} H_{i \ell_{1} \ell_{2}} \bar{H}_{\ell_{3} \ell_{4}}^{i}\right) \Gamma^{\ell_{1} \ell_{2} \ell_{3} \ell_{4}}\right) \eta_{-}\right\rangle \\
& +2 \operatorname{Re} \int_{\mathcal{S}}\left\langle\eta_{-},\left(\frac{7 i}{32} \Lambda^{i} \Phi_{i}+\frac{7}{64} h^{i} \Phi_{i}-\frac{11 i}{96} Y_{\ell_{1} \ell_{2} \ell_{3}} H^{\ell_{1} \ell_{2} \ell_{3}}\right.\right. \\
& +\left(-\frac{5 i}{192} \Lambda_{\ell_{1}} H_{\ell_{2} \ell_{3} \ell_{4}}-\frac{5}{384} h_{\ell_{1}} H_{\ell_{2} \ell_{3} \ell_{4}}\right. \\
& \left.\left.\left.+\frac{5 i}{96} \Phi_{\ell_{1}} Y_{\ell_{2} \ell_{3} \ell_{4}}+\frac{3 i}{64} Y_{i \ell_{1} \ell_{2}} H_{\ell_{3} \ell_{4}}^{i}\right) \Gamma^{\ell_{1} \ell_{2} \ell_{3} \ell_{4}}\right) C * \eta_{-}\right\rangle
\end{aligned}
$$

where we have made use of the identities

$$
\left\langle\eta_{-}, \Gamma_{\ell_{1} \ell_{2}} C * \eta_{-}\right\rangle=0, \quad\left\langle\eta_{-}, \Gamma_{\ell_{1} \ell_{2} \ell_{3} \ell_{4} \ell_{5} \ell_{6}} C * \eta_{-}\right\rangle=0 .
$$

It is also straightforward to evaluate $\mathcal{I}_{1}$, to obtain

$$
\begin{aligned}
\mathcal{I}_{1} & =\int_{\mathcal{S}}-\tilde{\nabla}_{i}\left\langle\eta_{-}, \Gamma^{i j} \tilde{\nabla}_{j} \eta_{-}\right\rangle-\frac{1}{4} \int_{\mathcal{S}} h^{i} \tilde{\nabla}_{i}\left\langle\eta_{-}, \eta_{-}\right\rangle \\
& +\int_{\mathcal{S}}\left\langle\eta_{-},\left(-\frac{1}{8} h^{2}-\frac{1}{3} Y_{\ell_{1} \ell_{2} \ell_{3}} Y^{\ell_{1} \ell_{2} \ell_{3}}-\frac{1}{8} \Phi_{i} \bar{\Phi}^{i}\right.\right. \\
& \left.\left.-\frac{1}{2} \xi_{i} \bar{\xi}^{i}-\frac{1}{48} H_{\ell_{1} \ell_{2} \ell_{3}} \bar{H}^{\ell_{1} \ell_{2} \ell_{3}}\right) \eta_{-}\right\rangle,
\end{aligned}
$$

where we have used the Einstein equations (A.8) to compute

$$
\tilde{R}=-\tilde{\nabla}^{i} h_{i}+\frac{1}{2} h^{2}+\frac{4}{3} Y_{\ell_{1} \ell_{2} \ell_{3}} Y^{\ell_{1} \ell_{2} \ell_{3}}+\frac{1}{2} \Phi_{i} \bar{\Phi}^{i}+2 \xi_{i} \bar{\xi}^{i}+\frac{1}{12} H_{\ell_{1} \ell_{2} \ell_{3}} \bar{H}^{\ell_{1} \ell_{2} \ell_{3}},
$$

and we recall

$$
\Gamma^{i j} \tilde{\nabla}_{i} \tilde{\nabla}_{j} \eta_{-}=-\frac{1}{4} \tilde{R} \eta_{-} .
$$

It remains to compute $\mathcal{I}_{2}$. First note that 


$$
\begin{aligned}
\mathcal{I}_{2} & =\int_{\mathcal{S}} \tilde{\nabla}_{i}\left\langle\eta_{-}, \frac{i}{2} \Gamma^{i j} \Lambda_{j} \eta_{-}\right\rangle \\
& +\int_{\mathcal{S}}\left\langle\eta_{-},\left(-\tilde{\nabla}_{i} \psi^{(-) i}+\tilde{\nabla}_{i}\left(\Gamma^{i} \psi^{(-)}\right)\right) \eta_{-}\right\rangle \\
& +\int_{\mathcal{S}}\left\langle\eta_{-},\left(\left(\psi^{(-) i}\right)^{\dagger}-\psi^{(-) i}-\left(\psi^{(-) \dagger}-\psi^{(-)}\right) \Gamma^{i}\right) \tilde{\nabla}_{i} \eta_{-}\right\rangle \\
& +\int_{\mathcal{S}}\left\langle\eta_{-},\left(\Gamma^{i} \psi^{(-)}-\psi^{(-)} \Gamma^{i}\right) \tilde{\nabla}_{i} \eta_{-}\right\rangle \\
& +\operatorname{Re} \int_{\mathcal{S}}\left\langle\eta_{-},\left(-2 \tilde{\nabla}_{i} \theta^{(-) i}+2 \tilde{\nabla}_{i}\left(\Gamma^{i} \theta^{(-)}\right)\right) C * \eta_{-}+C *\left(\left(\left(\tilde{\nabla}_{i} \theta^{(-) i}\right)^{\dagger}-\tilde{\nabla}_{i}\left(\Gamma^{i} \theta^{(-)}\right)^{\dagger}\right) \eta_{-}\right)\right\rangle \\
& +\operatorname{Re} \int_{\mathcal{S}}\left\langle\eta_{-},\left(-\theta^{(-) i}+\Gamma^{i} \theta^{(-)}\right) \tilde{\nabla}_{i} C * \eta_{-}+C *\left(\left(\left(\theta^{(-) i}\right)^{\dagger}-\left(\Gamma^{i} \theta^{(-)}\right)^{\dagger}\right) \tilde{\nabla}_{i} \eta_{-}\right)\right\rangle . \quad(\mathrm{C} .34)
\end{aligned}
$$

In order to evaluate this expression, it is useful to note that

$\operatorname{Re}\left(\int_{\mathcal{S}}\left\langle\eta_{-},\left(-\tilde{\nabla}_{i} \psi^{(-) i}+\tilde{\nabla}_{i}\left(\Gamma^{i} \psi^{(-)}\right)\right) \eta_{-}\right\rangle\right)=\int_{\mathcal{S}}\left\langle\eta_{-},\left(-\frac{i}{4} d \Lambda_{\ell_{1} \ell_{2}} \Gamma^{\ell_{1} \ell_{2}}-\frac{3 i}{4} \tilde{\nabla}^{i} Y_{i \ell_{1} \ell_{2}} \Gamma^{\ell_{1} \ell_{2}}\right) \eta_{-}\right\rangle$,

where, as a consequence of (A.3),

$$
\left\langle\eta_{-}, d \Lambda_{\ell_{1} \ell_{2}} \Gamma^{\ell_{1} \ell_{2}} \eta_{-}\right\rangle=-2 i\left\langle\bar{\xi}_{i} \Gamma^{i} \eta_{-}, \bar{\xi}_{j} \Gamma^{j} \eta_{-}\right\rangle+2 i\left\langle\eta_{-}, \xi^{i} \bar{\xi}_{i} \eta_{-}\right\rangle
$$

Also, one has

$$
\begin{array}{r}
\int_{\mathcal{S}}\left\langle\eta_{-},\left(\left(\psi^{(-) i}\right)^{\dagger}-\psi^{(-) i}-\left(\psi^{(-) \dagger}-\psi^{(-)}\right) \Gamma^{i}\right) \tilde{\nabla}_{i} \eta_{-}\right\rangle+\int_{\mathcal{S}}\left\langle\eta_{-},\left(\Gamma^{i} \psi^{(-)}-\psi^{(-)} \Gamma^{i}\right) \tilde{\nabla}_{i} \eta_{-}\right\rangle \\
=\int_{\mathcal{S}}\left\langle\eta_{-},\left(\left(-\frac{1}{2} h_{j} \Gamma^{j}+\frac{i}{6} Y_{\ell_{1} \ell_{2} \ell_{3}} \Gamma^{\ell_{1} \ell_{2} \ell_{3}}\right) \Gamma^{i}+\frac{1}{2} h^{i}-\frac{i}{2} Y^{i} \ell_{1} \ell_{2}\right) \tilde{\nabla}_{i} \eta_{-}\right\rangle
\end{array}
$$

with

$$
\operatorname{Re} \int_{\mathcal{S}}\left\langle\eta_{-}, h^{i} \tilde{\nabla}_{i} \eta_{-}\right\rangle=\frac{1}{2} \int_{\mathcal{S}} h^{i} \tilde{\nabla}_{i}\left\langle\eta_{-}, \eta_{-}\right\rangle
$$

and

$$
\operatorname{Re} \int_{\mathcal{S}}\left\langle\eta_{-}, i Y^{i} \ell_{1} \ell_{2} \Gamma^{\ell_{1} \ell_{2}} \tilde{\nabla}_{i} \eta_{-}\right\rangle=-\frac{1}{2} \int_{\mathcal{S}}\left\langle\eta_{-}, i \tilde{\nabla}^{i} Y_{i \ell_{1} \ell_{2}} \Gamma^{\ell_{1} \ell_{2}} \eta_{-}\right\rangle
$$

and hence (C.37) implies

$$
\begin{array}{r}
\operatorname{Re}\left(\int_{\mathcal{S}}\left\langle\eta_{-},\left(\left(\psi^{(-) i}\right)^{\dagger}-\psi^{(-) i}-\left(\psi^{(-) \dagger}-\psi^{(-)}\right) \Gamma^{i}\right) \tilde{\nabla}_{i} \eta_{-}\right\rangle+\int_{\mathcal{S}}\left\langle\eta_{-},\left(\Gamma^{i} \psi^{(-)}-\psi^{(-)} \Gamma^{i}\right) \tilde{\nabla}_{i} \eta_{-}\right\rangle\right) \\
=\operatorname{Re} \int_{\mathcal{S}}\left\langle\eta_{-},\left(-\frac{1}{2} h_{j} \Gamma^{j}+\frac{i}{6} Y_{\ell_{1} \ell_{2} \ell_{3}} \Gamma^{\ell_{1} \ell_{2} \ell_{3}}\right) \Gamma^{i} \tilde{\nabla}_{i} \eta_{-}\right\rangle+\frac{1}{4} \int_{\mathcal{S}} h^{i} \tilde{\nabla}_{i}\left\langle\eta_{-}, \eta_{-}\right\rangle \\
+\frac{1}{4} \int_{\mathcal{S}}\left\langle\eta_{-}, i \tilde{\nabla}^{i} Y_{i \ell_{1} \ell_{2}} \Gamma^{\ell_{1} \ell_{2}} \eta_{-}\right\rangle
\end{array}
$$


In addition, one has

$$
\begin{array}{r}
\operatorname{Re} \int_{\mathcal{S}}\left\langle\eta_{-},\left(-2 \tilde{\nabla}_{i} \theta^{(-) i}+2 \tilde{\nabla}_{i}\left(\Gamma^{i} \theta^{(-)}\right)\right) C * \eta_{-}+C *\left(\left(\left(\tilde{\nabla}_{i} \theta^{(-) i}\right)^{\dagger}-\tilde{\nabla}_{i}\left(\Gamma^{i} \theta^{(-)}\right)^{\dagger}\right) \eta_{-}\right)\right\rangle \\
=\operatorname{Re} \int_{\mathcal{S}}\left\langle\eta_{-},\left(-\frac{7}{16} \tilde{\nabla}^{i} \Phi_{i}+\frac{5}{384} d H_{\ell_{1} \ell_{2} \ell_{3} \ell_{4}} \Gamma^{\ell_{1} \ell_{2} \ell_{3} \ell_{4}}\right) C * \eta_{-}\right\rangle .
\end{array}
$$

Also,

$$
\begin{array}{r}
\operatorname{Re} \int_{\mathcal{S}}\left\langle\eta_{-},\left(-\theta^{(-) i}+\Gamma^{i} \theta^{(-)}\right) \tilde{\nabla}_{i} C * \eta_{-}+C *\left(\left(\left(\theta^{(-)} i\right)^{\dagger}-\left(\Gamma^{i} \theta^{(-)}\right)^{\dagger}\right) \tilde{\nabla}_{i} \eta_{-}\right)\right\rangle \\
=\operatorname{Re} \int_{\mathcal{S}}\left\langle\eta_{-},\left(\frac{3}{8} \Gamma^{j} \Phi_{j}+\frac{1}{48} H_{\ell_{1} \ell_{2} \ell_{3}} \Gamma^{\ell_{1} \ell_{2} \ell_{3}}\right) \Gamma^{i} \tilde{\nabla}_{i} C * \eta_{-}\right. \\
\left.-\frac{3}{8} \Phi^{i} \tilde{\nabla}_{i} C * \eta_{-}+\frac{1}{48} \Gamma^{i \ell_{1} \ell_{2} \ell_{3}} H_{\ell_{1} \ell_{2} \ell_{3}} \tilde{\nabla}_{i} C * \eta_{-}\right\rangle
\end{array}
$$

where

$$
\operatorname{Re} \int_{\mathcal{S}}\left\langle\eta_{-}, \Phi^{i} \tilde{\nabla}_{i} C * \eta_{-}\right\rangle=-\frac{1}{2} \operatorname{Re} \int_{\mathcal{S}}\left\langle\eta_{-},\left(\tilde{\nabla}^{i} \Phi_{i}\right) C * \eta_{-}\right\rangle
$$

and

$$
\operatorname{Re} \int_{\mathcal{S}}\left\langle\eta_{-}, \Gamma^{i \ell_{1} \ell_{2} \ell_{3}} H_{\ell_{1} \ell_{2} \ell_{3}} \tilde{\nabla}_{i} C * \eta_{-}\right\rangle=-\frac{1}{8} \operatorname{Re} \int_{\mathcal{S}}\left\langle\eta_{-}, d H_{\ell_{1} \ell_{2} \ell_{3} \ell_{4}} \Gamma^{\ell_{1} \ell_{2} \ell_{3} \ell_{4}} C * \eta_{-}\right\rangle
$$

and we remark that the surface terms obtained on integrating by parts in (C.43) and (C.44) vanish.

It follows that (C.42) can be rewritten as

$$
\begin{array}{r}
\operatorname{Re} \int_{\mathcal{S}}\left\langle\eta_{-},\left(-\theta^{(-) i}+\Gamma^{i} \theta^{(-)}\right) \tilde{\nabla}_{i} C * \eta_{-}+C *\left(\left(\left(\theta^{(-) i}\right)^{\dagger}-\left(\Gamma^{i} \theta^{(-)}\right)^{\dagger}\right) \tilde{\nabla}_{i} \eta_{-}\right)\right\rangle \\
=\operatorname{Re} \int_{\mathcal{S}}\left\langle\eta_{-},\left(\frac{3}{8} \Gamma^{j} \Phi_{j}+\frac{1}{48} H_{\ell_{1} \ell_{2} \ell_{3}} \Gamma^{\ell_{1} \ell_{2} \ell_{3}}\right) \Gamma^{i} \tilde{\nabla}_{i} C * \eta_{-}\right\rangle \\
+\operatorname{Re} \int_{\mathcal{S}}\left\langle\eta_{-},\left(\frac{3}{16} \tilde{\nabla}^{i} \Phi_{i}-\frac{1}{384} d H_{\ell_{1} \ell_{2} \ell_{3} \ell_{4}} \Gamma^{\ell_{1} \ell_{2} \ell_{3} \ell_{4}}\right) C * \eta_{-}\right\rangle
\end{array}
$$

On substituting (C.35), (C.40), (C.41) and (C.45) into (C.34), one obtains, after using the bosonic Bianchi identities/field equations, 


$$
\begin{aligned}
\mathcal{I}_{2} & =\int_{\mathcal{S}} \tilde{\nabla}_{i}\left\langle\eta_{-}, \frac{i}{2} \Gamma^{i j} \Lambda_{j} \eta_{-}\right\rangle+\frac{1}{2}\left\langle\eta_{-}, \xi_{i} \bar{\xi}^{i} \eta_{-}\right\rangle \\
& +\int_{\mathcal{S}}-\frac{1}{2}\left\langle\bar{\xi}_{i} \Gamma^{i} \eta_{-}, \bar{\xi}_{j} \Gamma^{j} \eta_{-}\right\rangle+\frac{1}{4} \int_{\mathcal{S}} h^{i} \tilde{\nabla}_{i}\left\langle\eta_{-}, \eta_{-}\right\rangle \\
& +\int_{\mathcal{S}}\left\langle\eta_{-},-\frac{1}{576} \epsilon_{\ell_{1} \ell_{2}}^{q_{1} q_{2} q_{3} q_{4} q_{5} q_{6}} H_{q_{1} q_{2} q_{3}} \bar{H}_{q_{4} q_{5} q_{6}} \Gamma^{\ell_{1} \ell_{2}} \eta_{-}\right\rangle \\
& +\operatorname{Re} \int_{\mathcal{S}}\left\langle\eta_{-},\left(-\frac{1}{2} h_{j} \Gamma^{j}+\frac{i}{6} Y_{\ell_{1} \ell_{2} \ell_{3}} \Gamma^{\ell_{1} \ell_{2} \ell_{3}}\right) \Gamma^{i} \tilde{\nabla}_{i} \eta_{-}\right\rangle \\
& +\operatorname{Re} \int_{\mathcal{S}}\left\langle\eta_{-},\left(\frac{3}{8} \Phi_{j} \Gamma^{j}+\frac{1}{48} H_{\ell_{1} \ell_{2} \ell_{3}} \Gamma^{\ell_{1} \ell_{2} \ell_{3}}\right) \Gamma^{i} \tilde{\nabla}_{i} C * \eta_{-}\right\rangle \\
& +\operatorname{Re} \int_{\mathcal{S}}\left\langle\eta_{-}, \frac{1}{4}\left(-i \Lambda^{i} \Phi_{i}-\xi^{i} \bar{\Phi}_{i}+\frac{2 i}{3} H_{\ell_{1} \ell_{2} \ell_{3}} Y^{\ell_{1} \ell_{2} \ell_{3}}\right) C * \eta_{-}\right\rangle \\
& +\operatorname{Re} \int_{\mathcal{S}}\left\langle\eta_{-}, \frac{1}{96}\left(4 i \Lambda_{\ell_{1}} H_{\ell_{2} \ell_{3} \ell_{4}}-4 \xi_{\ell_{1}} \bar{H}_{\ell_{2} \ell_{3} \ell_{4}}\right) \Gamma^{\ell_{1} \ell_{2} \ell_{3} \ell_{4}} C * \eta_{-}\right\rangle
\end{aligned}
$$

To proceed further, complete the square involving the $\xi$-terms in lines 2, 6 and 7 of the above expression. This produces a term proportional to the norm squared of the LHS of the algebraic condition (2.17), together with a number of counterterms. Also rewrite lines 4 and 5 in terms of the horizon Dirac equation (C.19) and its conjugate (with respect to $C *)$; again there are a number of algebraic counterterms. On performing these calculations, one obtains

$$
\begin{aligned}
& \mathcal{I}_{2}=\int_{\mathcal{S}} \tilde{\nabla}_{i}\left\langle\eta_{-}, \frac{i}{2} \Gamma^{i j} \Lambda_{j} \eta_{-}\right\rangle+\frac{1}{4} \int_{\mathcal{S}} h^{i} \tilde{\nabla}_{i}\left\langle\eta_{-}, \eta_{-}\right\rangle \\
& +\int_{\mathcal{S}}-\frac{1}{2}\left\langle\bar{\xi}_{i} \Gamma^{i} \eta_{-}+\left(\frac{1}{4} \bar{\Phi}_{i} \Gamma^{i}+\frac{1}{24} \bar{H}_{\ell_{1} \ell_{2} \ell_{3}} \Gamma^{\ell_{1} \ell_{2} \ell_{3}}\right) C * \eta_{-},\right. \\
& \left.\bar{\xi}_{j} \Gamma^{j} \eta_{-}+\left(\frac{1}{4} \bar{\Phi}_{j} \Gamma^{j}+\frac{1}{24} \bar{H}_{q_{1} q_{2} q_{3}} \Gamma^{q_{1} q_{2} q_{3}}\right) C * \eta_{-}\right\rangle \\
& +\operatorname{Re} \int_{\mathcal{S}}\left\langle\eta_{-},\left(-\frac{1}{2} h_{j} \Gamma^{j}+\frac{i}{6} Y_{\ell_{1} \ell_{2} \ell_{3}} \Gamma^{\ell_{1} \ell_{2} \ell_{3}}\right)\left(\Gamma^{i} \tilde{\nabla}_{i} \eta_{-}+\psi^{(-)} \eta_{-}+\theta^{(-)} C * \eta_{-}\right)\right\rangle \\
& +\operatorname{Re} \int_{\mathcal{S}}\left\langle\eta_{-},\left(\frac{3}{8} \Phi_{j} \Gamma^{j}+\frac{1}{48} H_{\ell_{1} \ell_{2} \ell_{3}} \Gamma^{\ell_{1} \ell_{2} \ell_{3}}\right) C *\left(\Gamma^{i} \tilde{\nabla}_{i} \eta_{-}+\psi^{(-)} \eta_{-}+\theta^{(-)} C * \eta_{-}\right)\right\rangle \\
& +\int_{\mathcal{S}}\left\langle\eta_{-},\left(\frac{1}{2} \xi_{i} \bar{\xi}_{i}+\frac{1}{8} h^{2}+\frac{1}{6} Y_{\ell_{1} \ell_{2} \ell_{3}} Y^{\ell_{1} \ell_{2} \ell_{3}}+\frac{1}{8} \Phi_{i} \bar{\Phi}^{i}+\frac{1}{96} H_{\ell_{1} \ell_{2} \ell_{3}} \bar{H}^{\ell_{1} \ell_{2} \ell_{3}}\right.\right. \\
& +\left(\frac{i}{4} \Lambda_{\ell_{1}} h_{\ell_{2}}+\frac{3 i}{8} h^{i} Y_{i \ell_{1} \ell_{2}}+\frac{1}{16} \Phi_{\ell_{1}} \bar{\Phi}_{\ell_{2}}-\frac{3}{64} \Phi^{i} \bar{H}_{i \ell_{1} \ell_{2}}+\frac{3}{64} \bar{\Phi}^{i} H_{i \ell_{1} \ell_{2}}\right. \\
& \left.-\frac{1}{576} \epsilon_{\ell_{1} \ell_{2}}{ }^{q_{1} q_{2} q_{3} q_{4} q_{5} q_{6}} H_{q_{1} q_{2} q_{3}} \bar{H}_{q_{4} q_{5} q_{6}}\right) \Gamma^{\ell_{1} \ell_{2}} \\
& +\left(\frac{1}{12} \Lambda_{\ell_{1}} Y_{\ell_{2} \ell_{3} \ell_{4}}-\frac{1}{4} Y^{i}{ }_{\ell_{1} \ell_{2}} Y_{i \ell_{3} \ell_{4}}-\frac{1}{192} \Phi_{\ell_{1}} \bar{H}_{\ell_{2} \ell_{3} \ell_{4}}-\frac{1}{192} \bar{\Phi}_{\ell_{1}} H_{\ell_{2} \ell_{3} \ell_{4}}\right. \\
& \left.\left.\left.-\frac{1}{64} H^{i}{ }_{\ell_{1} \ell_{2}} \bar{H}_{i \ell_{3} \ell_{4}}\right) \Gamma^{\ell_{1} \ell_{2} \ell_{3} \ell_{4}}\right) \eta_{-}\right\rangle \\
& +\operatorname{Re} \int_{\mathcal{S}}\left\langle\eta_{-},\left(-\frac{7 i}{16} \Lambda^{i} \Phi_{i}-\frac{7}{32} h_{i} \Phi^{i}+\frac{11 i}{48} Y_{\ell_{1} \ell_{2} \ell_{3}} H^{\ell_{1} \ell_{2} \ell_{3}}\right.\right.
\end{aligned}
$$




$$
\left.\left.+\left(\frac{5 i}{96} \Lambda_{\ell_{1}} H_{\ell_{2} \ell_{3} \ell_{4}}+\frac{5}{192} h_{\ell_{1}} H_{\ell_{2} \ell_{3} \ell_{4}}-\frac{5 i}{48} \Phi_{\ell_{1}} Y_{\ell_{2} \ell_{3} \ell_{4}}-\frac{3 i}{32} Y_{\ell_{1} \ell_{2}}^{i} H_{i \ell_{3} \ell_{4}}\right) \Gamma^{\ell_{1} \ell_{2} \ell_{3} \ell_{4}}\right) \eta_{-}\right\rangle .
$$

On combining (C.47) with (C.31) and (C.29), one obtains

$$
\begin{aligned}
\mathcal{I}= & -\frac{1}{2} \int_{\mathcal{S}}\left\|\mathcal{A}^{(-)} \eta_{-}\right\|^{2}+\operatorname{Re} \int_{\mathcal{S}}\left\langle\eta_{-},\left(-\frac{1}{2} h_{j} \Gamma^{j}+\frac{i}{6} Y_{\ell_{1} \ell_{2} \ell_{3}} \Gamma^{\ell_{1} \ell_{2} \ell_{3}}\right) \mathcal{D}^{(-)} \eta_{-}\right\rangle \\
& +\operatorname{Re} \int_{\mathcal{S}}\left\langle\eta_{-},\left(\frac{3}{8} \Phi_{j} \Gamma^{j}+\frac{1}{48} H_{\ell_{1} \ell_{2} \ell_{3}} \Gamma^{\ell_{1} \ell_{2} \ell_{3}}\right) C * \mathcal{D}^{(-)} \eta_{-}\right\rangle
\end{aligned}
$$

where we have made use of the identity

$$
\int_{\mathcal{S}} \tilde{\nabla}_{i}\left\langle\eta_{-}, \Gamma^{i j}\left(-\tilde{\nabla}_{j} \eta_{-}+\frac{i}{2} \Lambda_{j} \eta_{-}\right)\right\rangle=0
$$

The expression (C.48) establishes the Lichnerowicz identity. Suppose then that we impose the horizon Dirac equation (C.19). Then (C.48) implies that

$$
\int_{\mathcal{S}}\left\|\nabla^{(-)} \eta_{-}\right\|^{2}=-\frac{1}{2} \int_{\mathcal{S}}\left\|\mathcal{A}^{(-)} \eta_{-}\right\|^{2}
$$

As the LHS is non-negative, whereas the RHS is non-positive, both sides must vanish. The vanishing of the LHS implies the horizon KSE (2.14), and the vanishing of the RHS implies (2.17).

\section{Appendix D Proof of the preservation of fluxes by Killing vectors}

In this appendix we will give a short proof of the fact that the vector fields constructed as bi-linears of Killing spinors preserve all fields of the theory. This is a consequence of the results of [23], and a concise proof in the string frame has been given in [28]. Here we shall outline a proof in the Einstein frame for completeness. This relies only on the application of the KSEs (2.3) and (2.4) and thus holds in general.

It is convenient to introduce the following notation

$$
\begin{aligned}
& \alpha_{B_{1} \cdots B_{k}}^{I J} \equiv B\left(\epsilon^{I}, \Gamma_{B_{1} \cdots B_{k}} \epsilon^{J}\right), \\
& \sigma_{B_{1} \cdots B_{k}}^{I J} \equiv B\left(C * \epsilon^{I}, \Gamma_{B_{1} \cdots B_{k}} C * \epsilon^{J}\right), \\
& \tau_{B_{1} \cdots B_{k}}^{I J} \equiv B\left(\epsilon^{I}, \Gamma_{B_{1} \cdots B_{k}} C * \epsilon^{J}\right),
\end{aligned}
$$

where the inner product $B\left(\epsilon^{I}, \epsilon^{J}\right) \equiv\left\langle\Gamma_{0} C * \epsilon^{I}, \epsilon^{J}\right\rangle$ is antisymmetric, i.e. $B\left(\epsilon^{I}, \epsilon^{J}\right)=$ $-B\left(\epsilon^{J}, \epsilon^{I}\right)$ and all $\Gamma$-matrices are anti-Hermitian with respect to this inner product, i.e. $B\left(\Gamma_{A} \epsilon^{I}, \epsilon^{J}\right)=-B\left(\epsilon^{I}, \Gamma_{A} \epsilon^{J}\right)$. See [23] for the conventions.

Denoting $\alpha_{B_{1} \cdots B_{k}}^{I J}=\alpha_{(k)}^{I J}$ the bilinears have the symmetry properties

$$
\begin{gathered}
\alpha_{(k)}^{I J}=\alpha_{(k)}^{J I}, \quad \sigma_{(k)}^{I J}=\sigma_{(k)}^{J I} \quad k=1,2,5, \\
\alpha_{(k)}^{I J}=-\alpha_{(k)}^{J I}, \quad \sigma_{(k)}^{I J}=-\sigma_{(k)}^{J I} \quad k=0,3,4,
\end{gathered}
$$


and their complex conjugates satisfy the following relations

$$
\begin{array}{ll}
\bar{\alpha}_{(k)}^{I J}=\sigma_{(k)}^{I J}, & \\
\bar{\tau}_{(k)}^{I J}=-\tau_{(k)}^{J I} & k=1,2,5, \\
\bar{\tau}_{(k)}^{I J}=\tau_{(k)}^{J I} & k=0,3,4 .
\end{array}
$$

First we verify that there is a 1-form bi-linear whose associated vector is Killing. We write the gravitino KSE as

$$
\left(\nabla_{A}+\Sigma_{A}\right) \epsilon=0
$$

where

$$
\begin{aligned}
\Sigma_{A}=- & \frac{i}{2} Q_{A}+\frac{i}{48} F_{A C_{1} C_{2} C_{3} C_{4}} \Gamma^{C_{1} C_{2} C_{3} C_{4}} \\
& \quad-\frac{1}{96}\left(G_{C_{1} C_{2} C_{3}} \Gamma_{A} C_{1} C_{2} C_{3}-9 G_{A C_{1} C_{2}} \Gamma^{C_{1} C_{2}}\right) C *
\end{aligned}
$$

which we use to replace covariant derivatives with fluxes and $\Gamma$-matrices. The 1 -form bilinear associated with the Killing vector is $\tau_{A}^{(I J)} e^{A}$, which we see by computing

$$
\begin{aligned}
\nabla_{A} \tau_{B}^{(I J)} & =\nabla_{A} B\left(\epsilon^{(I}, \Gamma_{B} C * \epsilon^{J)}\right) \\
& =B\left(\nabla_{A} \epsilon^{(I}, \Gamma_{B} C * \epsilon^{J)}\right)+B\left(\epsilon^{(I}, \Gamma_{B} C * \nabla_{A} \epsilon^{J)}\right) \\
& =-B\left(\Sigma_{A} \epsilon^{(I}, \Gamma_{B} C * \epsilon^{J)}\right)-B\left(\epsilon^{(I}, \Gamma_{B} C * \Sigma_{A} \epsilon^{J)}\right) \\
& =B\left(\Gamma_{B} C * \epsilon^{(I}, \Sigma_{A} \epsilon^{J)}\right)-B\left(\epsilon^{(I}, \Gamma_{B} C * \Sigma_{A} \epsilon^{J)}\right) \\
& =-\bar{B}\left(\epsilon^{(I}, C * \Gamma_{B} \Sigma_{A} \epsilon^{J)}\right)-B\left(\epsilon^{(I}, \Gamma_{B} C * \Sigma_{A} \epsilon^{J)}\right) \\
& =-2 \operatorname{Re} B\left(\epsilon^{(I}, C * \Gamma_{B} \Sigma_{A} \epsilon^{J)}\right) \\
& =\operatorname{Re}\left(-\frac{3}{8} G_{A B}{ }^{C} \bar{\alpha}_{C}^{I J}-\frac{1}{48} G^{C_{1} C_{2} C_{3}} \bar{\alpha}_{A B C_{1} C_{2} C_{3}}^{I J}+\frac{i}{6} F_{A B}{ }^{C_{1} C_{2} C_{3}} \tau_{C_{1} C_{2} C_{3}}^{I J}\right)
\end{aligned}
$$

Since the resulting expression is antisymmetric in its free indices we find that $\nabla_{(A} \tau_{B)}^{(I J)}=0$ and hence the vector associated with $\tau_{A}^{(I J)} e^{A}$ is Killing.

Note that the dilatino KSE (2.4)

$$
\mathcal{A} \epsilon \equiv\left(P_{A} \Gamma^{A}(C *)+\frac{1}{24} G_{A_{1} A_{2} A_{3}} \Gamma^{A_{1} A_{2} A_{3}}\right) \epsilon=0
$$

implies that

$$
0=B\left(\epsilon^{(I}, \mathcal{A} \epsilon^{J)}\right)=P^{A} \tau_{A}^{(I J)}
$$

and hence $i_{K} P=0$, where $K=\tau_{A}^{(I J)} e^{A}$ denotes the 1-form associated with the Killing vector. With this relation it follows that the Killing vector leaves $P$ and $d Q$ invariant up to a $U(1)$ transformation:

$$
\mathcal{L}_{K} P=i_{K} d P+d\left(i_{K} P\right)=2 i i_{K}(Q \wedge P)=2 i\left(i_{K} Q\right) P
$$


and

$$
\mathcal{L}_{K} d Q=d i_{k} d Q=-i d i_{K}(P \wedge \bar{P})=0,
$$

where we have used the Bianchi identities for $P$ and $Q$

$$
d P=2 i Q \wedge P, \quad d Q=-i P \wedge \bar{P} .
$$

To see that the 3 -form flux $G$ is preserved we need to analyse the 1-form bi-linear which is not related to the Killing vector, i.e. $\alpha_{A}^{I J} e^{A}$. As above, we find that ${ }^{12}$

$$
\begin{aligned}
\nabla_{[A} \alpha_{B]}^{I J}= & -2 B\left(\epsilon^{(I}, \Gamma_{B} \Sigma_{A} \epsilon^{J)}\right) \\
= & -\frac{1}{2} G_{A B}{ }^{C} \tau_{C}^{(I J)}+i Q_{[A} \alpha_{B]}^{I J}-P_{[A} \bar{\alpha}_{B]}^{I J},
\end{aligned}
$$

or equivalently

$$
d \alpha=-i_{K} G+i Q \wedge \alpha-P \wedge \bar{\alpha}
$$

where we have suppressed the indices labelling the Killing spinors on $\alpha$. To arrive at (D.12) we have used the dilatino KSE in the form

$$
B\left(C * \epsilon^{I}, \Gamma_{A B} \mathcal{A} \epsilon^{J}\right)
$$

to cancel bi-linears other than $\alpha$ and the 1-form bi-linear associated with the Killing vector. By taking the exterior derivative of (D.13), and resubstituting the expression for $d \alpha$, it follows that

$$
\mathcal{L}_{K} G=i\left(i_{K} Q\right) G
$$

where we have used the Bianchi identity for $G$

$$
d G=i Q \wedge G-P \wedge \bar{G}
$$

In now remains to verify that also the 5-form flux $F$ is preserved. Since we have already analyzed the possible 1 -form bi-linears, we proceed to the 3 -form bi-linears. There is only one 3-form bi-lilnear which is symmetric under the exchange of spinors, namely $\tau_{B_{1} B_{2} B_{3}}^{(I J)} e^{B_{1}} \wedge e^{B_{2}} \wedge e^{B_{3}}$. As above, we find

$$
d \tau=-4 i_{K} F+\frac{i}{2} G \wedge \bar{\alpha}-\frac{i}{2} \bar{G} \wedge \alpha
$$

Taking the exterior derivative of this expression yields

$$
\mathcal{L}_{K} F=0
$$

where we have used the expression above for $d \alpha$ and the Bianchi identity for $G$ and $F$

$$
d F=\frac{i}{8} G \wedge \bar{G}
$$

For the computations in this appendix the Mathematica package GAMMA [30] has been used.

\footnotetext{
${ }^{12}$ The terms containing the 5 -form flux $F$ and a 5 -form bi-linear vanish due to the self-duality of $F$.
} 


\section{References}

[1] B. Carter, "Black Holes," edited by C. de Witt and B.S. de Witt, (Gordon and Breach, New York, 1973).

[2] G.W. Gibbons, in Supersymmetry, Supergravity and Related Topics, eds. F. del Aguila, J. A. de Azc'arraga and L.E. Ibanez, (World Scientific 1985).

[3] G. W. Gibbons and P. K. Townsend, "Vacuum interpolation in supergravity via super p-branes," Phys. Rev. Lett. 71 (1993) 3754 [hep-th/9307049].

[4] O. Aharony, S. S. Gubser, J. M. Maldacena, H. Ooguri and Y. Oz, "Large N field theories, string theory and gravity," Phys. Rept. 323 (2000) 183 [hep-th/9905111].

[5] A. Strominger, "AdS(2) quantum gravity and string theory," JHEP 9901 (1999) 007 [hep-th/9809027].

[6] A. Sen, "Quantum Entropy Function from $\operatorname{AdS}(2) / C F T(1)$ Correspondence," Int. J. Mod. Phys. A 24 (2009) 4225 [arXiv:0809.3304 [hep-th]].

[7] H. K. Kunduri and J. Lucietti, "Classification of near-horizon geometries of extremal black holes," arXiv:1306.2517 [hep-th].

[8] J. Grover, J. B. Gutowski, G. Papadopoulos and W. A. Sabra, "Index Theory and Supersymmetry of 5D Horizons," arXiv:1303.0853 [hep-th].

[9] J. B. Gutowski and G. Papadopoulos, "Index theory and dynamical symmetry enhancement of M-horizons," arXiv:1303.0869 [hep-th].

[10] M. F. Atiyah and I. M. Singer, "The Index of elliptic operators. 1" Annals Math. 87 (1968) 484.

[11] S. M. Salamon, "Quaternionic Kähler Manifolds," Invent. Math. 67, 143-171 (1982).

D.D. Joyce, "Compact Riemannian 8-manifolds with Holonomy Spin(7)," Invent. Math. 123, 507-552 (1996).

[12] U. Gran, J. Gutowski, G. Papadopoulos and D. Roest, "N=31 is not IIB," JHEP 0702 (2007) 044 [hep-th/0606049].

U. Gran, J. Gutowski, G. Papadopoulos and D. Roest, "IIB solutions with $N>28$ Killing spinors are maximally supersymmetric," JHEP 0712 (2007) 070 [arXiv:0710.1829 [hep-th]].

[13] U. Gran, J. Gutowski and G. Papadopoulos, "Classification of IIB backgrounds with 28 supersymmetries," JHEP 1001 (2010) 044 [arXiv:0902.3642 [hep-th]].

[14] I. Bena and R. Roiban, "Supergravity pp wave solutions with twenty eight supercharges and twenty four supercharges," Phys. Rev. D 67 (2003) 125014 [hepth/0206195]. 
[15] U. Gran, J. Gutowski and G. Papadopoulos, "IIB backgrounds with five-form flux," Nucl. Phys. B798 (2008) 36; [arXiv:0705.2208 [hep-th]].

[16] H. S. Reall, "Higher dimensional black holes and supersymmetry," Phys. Rev. D68 (2003) 024024; [hep-th/0211290].

[17] J. Gutowski and G. Papadopoulos, "Topology of supersymmetric N=1, D=4 supergravity horizons," JHEP 1011 (2010) 114 [arXiv:1006.4369 [hep-th]].

[18] U. Gran, J. Gutowski and G. Papadopoulos, "IIB horizons," arXiv:1304.6539 [hepth].

[19] J. H. Schwarz, P. C. West and , "Symmetries and Transformations of Chiral N=2 D=10 Supergravity," Phys. Lett. B 126 (1983) 301.

[20] J. H. Schwarz, "Covariant Field Equations of Chiral N=2 D=10 Supergravity," Nucl. Phys. B 226 (1983) 269.

[21] B. R. Greene, A. D. Shapere, C. Vafa and S. -T. Yau, "Stringy Cosmic Strings and Noncompact Calabi-Yau Manifolds," Nucl. Phys. B 337 (1990) 1.

[22] G. W. Gibbons, M. B. Green and M. J. Perry, "Instantons and seven-branes in type IIB superstring theory," Phys. Lett. B 370 (1996) 37 [hep-th/9511080].

[23] U. Gran, J. Gutowski and G. Papadopoulos, "The Spinorial geometry of supersymmetric IIb backgrounds," Class. Quant. Grav. 22 (2005) 2453 [hep-th/0501177].

[24] J. Gutowski and G. Papadopoulos, "Heterotic Black Horizons," JHEP 1007 (2010) 011 [arXiv:0912.3472 [hep-th]].

"Heterotic horizons, Monge-Ampere equation and del Pezzo surfaces," JHEP 1010 (2010) 084 [arXiv:1003.2864 [hep-th]].

[25] U. Gran, J. Gutowski and G. Papadopoulos, "IIB black hole horizons with five-form flux and KT geometry," JHEP 1105 (2011) 050 [arXiv:1101.1247 [hep-th]].

[26] M. Atiyah and F. Hirzebruch, "Spin-Manifolds and Group Actions," Essays on topology and related topics, Memoires dédiés à Georges de Rham, ed. A. haefliger and R. Narasimhan, Springer-Verlag, New-York-Berlin (1970), 18.

[27] U. Gran, J. Gutowski and G. Papadopoulos, "The spinorial geometry of supersymmetric IIB backgrounds," Class. Quant. Grav. 22, 2453 (2005) [arXiv:hepth/0501177].

[28] J. M. Figueroa-O'Farrill, E. Hackett-Jones and G. Moutsopoulos, "The Killing superalgebra of ten-dimensional supergravity backgrounds," Class. Quant. Grav. 24 (2007) 3291 [hep-th/0703192].

[29] U. Gran, G. Papadopoulos, D. Roest and P. Sloane, "Geometry of all supersymmetric type I backgrounds," JHEP 0708 (2007) 074 [hep-th/0703143 [HEP-TH]]. 
[30] U. Gran, "GAMMA: A Mathematica package for performing gamma matrix algebra and Fierz transformations in arbitrary dimensions," hep-th/0105086. 